\title{
Algebraic Properties of Quasihomogeneous and Separately Quasihomogeneous Toeplitz Operators on the Pluriharmonic Bergman Space
}

\author{
Hongyan Guan, ${ }^{1,2}$ Liu Liu, ${ }^{1}$ and Yufeng $\mathrm{Lu}^{1}$ \\ ${ }^{1}$ School of Mathematical Sciences, Dalian University of Technology, Dalian 116024, China \\ ${ }^{2}$ School of Mathematics and Systems Science, Shenyang Normal University, Shenyang 110034, China \\ Correspondence should be addressed to Hongyan Guan; guanhy8010@163.com
}

Received 6 June 2013; Revised 2 September 2013; Accepted 2 September 2013

Academic Editor: Józef Banaś

Copyright ( $\odot 2013$ Hongyan Guan et al. This is an open access article distributed under the Creative Commons Attribution License, which permits unrestricted use, distribution, and reproduction in any medium, provided the original work is properly cited.

We study some algebraic properties of Toeplitz operator with quasihomogeneous or separately quasihomogeneous symbol on the pluriharmonic Bergman space of the unit ball in $\mathbb{C}^{n}$. We determine when the product of two Toeplitz operators with certain separately quasi-homogeneous symbols is a Toeplitz operator. Next, we discuss the zero-product problem for several Toeplitz operators, one of whose symbols is separately quasihomogeneous and the others are quasi-homogeneous functions, and show that the zero-product problem for two Toeplitz operators has only a trivial solution if one of the symbols is separately quasihomogeneous and the other is arbitrary. Finally, we also characterize the commutativity of certain quasihomogeneous or separately quasihomogeneous Toeplitz operators.

\section{Introduction}

For $n \geq 1$, let $\mathbb{C}^{n}$ be the cartesian product of $n$ copies of $\mathbb{C}$. For any points $z=\left(z_{1}, z_{2}, \ldots, z_{n}\right)$ and $w=\left(w_{1}, w_{2}, \ldots, w_{n}\right)$ in $\mathbb{C}^{n}$, we use the notions $\langle z, w\rangle=z_{1} \overline{w_{1}}+z_{2} \overline{w_{2}}+\cdots+z_{n} \overline{w_{n}}$ and $|z|=\sqrt{\left|z_{1}\right|^{2}+\left|z_{2}\right|^{2}+\cdots+\left|z_{n}\right|^{2}}$ for the inner product and the associated Euclidean norm. Let $\mathbb{B}_{n}$ denote the open unit ball which consists of points $z \in \mathbb{C}^{n}$ with $|z|<1$ and let $\mathbb{S}_{n}$ denote the unit sphere. Let $d V$ be the normalized Lebesgue volume measure on $\mathbb{B}_{n}$ and let $d \sigma$ be the normalized surface area measure on $\mathbb{S}_{n} \cdot L^{2}\left(\mathbb{B}_{n}, d V\right)$ is the Hilbert space consisting of all Lebesgue square integrable functions on $\mathbb{B}_{n}$ with the inner product

$$
\langle f, g\rangle=\int_{\mathbb{B}_{n}} f(z) \overline{g(z)} d V(z) .
$$

The Bergman space $L_{a}^{2}\left(\mathbb{B}_{n}\right)$ is the closed subspace consisting of the analytic functions in $L^{2}\left(\mathbb{B}_{n}, d V\right)$. Let $P$ be the orthogonal projection from $L^{2}\left(\mathbb{B}_{n}, d V\right)$ onto $L_{a}^{2}\left(\mathbb{B}_{n}\right)$, then $P$ can be expressed by

$$
(P f)(z)=\left\langle f, K_{z}\right\rangle=\int_{\mathbb{B}_{n}} f(w) \frac{1}{(1-\langle z, w\rangle)^{n+1}} d V(w),
$$

where $K_{z}(w)=1 /(1-\langle w, z\rangle)^{n+1}$ is the Bergman reproducing kernel.

A function $f$ is said to be pluriharmonic if and only if $f$ satisfies that $D_{j} \bar{D}_{k} f=0 \quad(j, k=1,2, \ldots, n)$, where $D_{j}=$ $\partial / \partial z_{j}$ and $\bar{D}_{j}=\partial / \partial \bar{z}_{j}$ (see page 9 of [1]). The pluriharmonic Bergman space, denoted by $L_{h}^{2}\left(\mathbb{B}_{n}\right)$, is the closed subspace of $L^{2}\left(\mathbb{B}_{n}, d V\right)$ consisting of all the pluriharmonic functions on $\mathbb{B}_{n}$. It is well known that $L_{h}^{2}\left(\mathbb{B}_{n}\right)$ is also a Hilbert space. We will write $Q$ for the orthogonal projection from $L^{2}\left(\mathbb{B}_{n}, d V\right)$ onto $L_{h}^{2}\left(\mathbb{B}_{n}\right)$. It is easy to verify that each point evaluation is a 
bounded linear functional on $L_{h}^{2}\left(\mathbb{B}_{n}\right)$. It follows that $L_{h}^{2}\left(\mathbb{B}_{n}\right)$ is also a reproducing function space with reproducing kernel:

$$
\begin{gathered}
R_{z}(w)=K_{z}(w)+\overline{K_{z}(w)}-1, \quad z, w \in \mathbb{B}_{n}, \\
Q f(z)=P f(z)+\overline{P \bar{f}(z)}-P f(0), \quad f \in L^{2}\left(\mathbb{B}_{n}, d V\right) .
\end{gathered}
$$

For a function $\phi \in L^{\infty}\left(\mathbb{B}_{n}, d V\right)$, we define the Toeplitz operator $T_{\phi}: L_{h}^{2}\left(\mathbb{B}_{n}\right) \rightarrow L_{h}^{2}\left(\mathbb{B}_{n}\right)$ with symbol $\phi$ by

$$
T_{\phi}(f)=Q(\phi f), \quad f \in L_{h}^{2}\left(\mathbb{B}_{n}\right) .
$$

For product problem, on the Hardy space, Brown and Halmos [2] showed that if $f$ and $g$ are bounded functions on the unit circle, then $T_{f} T_{g}$ is another Toeplitz operator if and only if either $\bar{f}$ or $g$ is analytic. In the setting of the Bergman space, the condition that either $\bar{f}$ or $g$ is analytic is still sufficient, but it is no longer necessary. Ahern and Čučković [3] showed that a Brown-Halmos type result holds for Toeplitz operators with harmonic symbols on $L_{a}^{2}\left(\mathbb{B}_{n}\right)$. In [4], Ahern characterized when the product of two Toeplitz operators with harmonic symbols is a Toeplitz operator. Later in [5], Louhichi et al. gave the necessary and sufficient conditions for the product of two quasihomogeneous Toeplitz operators to be a Toeplitz operator. Recently, Dong and Zhou [6] characterized when the product of quasihomogeneous Toeplitz operators is a Toeplitz operator on the harmonic Bergman space of the unit disk.

The situation is more complicated on the Hardy, Bergman, and harmonic Bergman spaces of several complex variables. In 2003, Ding [7] discussed the product problem for two Toeplitz operators with bounded symbols on the Hardy space $H^{2}\left(\mathbb{D}^{n}\right)$. After that, Choe et al. [8] solved the product problem for pluriharmonic Toeplitz operators on the Bergman space of the polydisk. On the Bergman space of the unit ball, Zhou and Dong [9] determined when the product of two radial Toeplitz operators is a Toeplitz operator. Later in [10], they discussed the product problem for two separately quasihomogeneous Toeplitz operators. In Zhang and Lu's paper [11], they characterized the product problem for two Toeplitz operators with quasihomogeneous symbols. On the pluriharmonic Bergman space, Yang et al. [12] gave the necessary and sufficient conditions for the product of two radial Toeplitz operators to be a Toeplitz operator.

For zero-product problem, on the Hardy space, Brown and Halmos [2] proved that if $f, g \in L^{\infty}(\mathbb{T})$ such that $T_{f} T_{g}=0$, then one of the symbols must be the zero function. Motivated by this result, Guo [13] showed that $T_{\phi_{1}} T_{\phi_{2}} \cdots T_{\phi_{n}}=$ 0 implies that $\phi_{i}=0$ for some $i$, when $n=5$. After that, $\mathrm{Gu}$ [14] proved that for $n=6$, the result in [13] is also true. Recently, Aleman and Vukotić [15] completely solved the zero-product problem for several Toeplitz operators on the Hardy space. On the Bergman space of the unit disk, Ahern and Čučković [3] obtained that the result is analogous to that in [2] for the zero-product problem of two harmonic Toeplitz operators. Furthermore, they got that $T_{f} T_{g}=0$ implies $f=0$ or $g=0$, where $f$ is arbitrary bounded and $g$ is radial in [16]. In 2003, Cučković [17] proved that if $f \in L^{\infty}(\mathbb{D})$ such that $T_{f} T_{z^{j}-\bar{z}^{l}}=0$, where $j, l$ are both positive integers, then $f=0$. Later in [18], Louhichi et al. considered the zero-product problem for $f, g \in L^{\infty}(\mathbb{D}, d A)$ with $g=$ $\sum_{k=-\infty}^{N} e^{i k \theta} g_{k}$, where $g_{k}$ is a bounded radial function and $N$ is a positive integer. On the Bergman space of the unit ball, Dong and Zhou [10] investigated the zero-product problem of two Toeplitz operators, one of whose symbols is separately quasihomogeneous and the other is arbitrary bounded. Bauer and Vasilevski [19] considered the zero-product problem and a more general problem of zero finite sum of finite products of Toeplitz operators. Recently, Yang et al. [12] discussed the zero-product problem for several radial Toeplitz operators on the pluriharmonic Bergman space of the unit ball.

For commuting problem, Brown and Halmos [2] firstly considered the commutativity of two Toeplitz operators on the Hardy space. They showed that two bounded Toeplitz operators $T_{\phi}$ and $T_{\psi}$ commute if and only if (1) both $\phi$ and $\psi$ are analytic, (2) both $\phi$ and $\psi$ are coanalytic, or (3) one is a linear function of the other. On the Bergman space of the unit disk, Axler and Čučković [20] obtained that the same result is also true for Toeplitz operators with bounded harmonic symbols. In [21], Cučković and Rao used the Mellin transform to study the commutativity of two Toeplitz operators on $L_{a}^{2}(\mathbb{D})$ and described those operators which commute with $T_{e^{i p \theta_{r} m}}$ for $(p, m) \in \mathbb{N} \times \mathbb{N}$. Later in [22], Louhichi and Zakariasy characterized commuting Toeplitz operators on $L_{a}^{2}(\mathbb{D})$ with quasihomogeneous symbols. On the Bergman space of the unit ball, Zheng [23] studied commuting Toeplitz operators with pluriharmonic symbols. Recently, extending Vasilevski's results in [24, 25], Quiroga-Barranco and Vasilevski gave the description of many (geometrically defined) classes of commuting Toeplitz operators of the unit ball in [26, 27]. Zhou and Dong [9] studied the commuting problem for quasihomogeneous Toeplitz operators. In 2012, Dong and Zhou [28] and Louhichi and Zakariasy [29] characterized the commuting Toeplitz operators with radial or quasihomogeneous symbols on the harmonic Bergman space of the unit disk. In papers [19, 30-33], the authors studied the wide classes of (nongeometrically defined) commutative Banach algebras generated by Toeplitz operators of the Bergman spaces on the unit ball.

Motivated by recent results of the unit ball in $[9,10$, 12], in this paper, on the pluriharmonic space of the unit ball, we first characterize the product of two Toeplitz operators with certain separately quasihomogeneous symbols to be a Toeplitz operator. Next, we solve the zero-product problem for several Toeplitz operators when one of the symbols is separately quasihomogeneous and the others are quasihomogeneous and show that zero-product problem for two Toeplitz operators with certain symbols has only a trivial solution. At last, the commutativity of certain (separately) quasihomogeneous Toeplitz operators is also discussed. 


\section{Preliminaries}

Let $\mathbb{N}$ denote the set of all nonnegative integers. For any $\alpha=$ $\left(\alpha_{1}, \alpha_{2}, \ldots, \alpha_{n}\right) \in \mathbb{N}^{n}$, for any point $z=\left(z_{1}, z_{2}, \ldots, z_{n}\right) \in \mathbb{B}_{n}$, we write

$$
\begin{gathered}
|\alpha|=\alpha_{1}+\alpha_{2}+\cdots+\alpha_{n}, \\
\alpha !=\alpha_{1} ! \alpha_{2} !, \ldots, \alpha_{n} ! \\
z^{\alpha}=z_{1}^{\alpha_{1}} z_{2}^{\alpha_{2}}, \ldots, z_{n}^{\alpha_{n}} .
\end{gathered}
$$

For two multi-indexes $\alpha=\left(\alpha_{1}, \alpha_{2}, \ldots, \alpha_{n}\right), \beta=\left(\beta_{1}, \beta_{2}\right.$, $\left.\ldots, \beta_{n}\right) \in \mathbb{N}^{n}$, the notations $\alpha \geq \beta$ and $\alpha \perp \beta$ mean that $\alpha_{i} \geq \beta_{i}$ for every $i$ and $\alpha_{1} \beta_{1}+\alpha_{2} \beta_{2}+\cdots+\alpha_{n} \beta_{n}=0$. Let $\alpha-\beta$ denote $\left(\alpha_{1}-\beta_{1}, \alpha_{2}-\beta_{2}, \ldots, \alpha_{n}-\beta_{n}\right)$. Moreover, if $\alpha \geq \beta$, $|\alpha-\beta|=|\alpha|-|\beta|$.

For a function $\phi \in L^{1}\left(\mathbb{B}_{n}, d V\right)$, $\phi$ is said to be radial if and only if $\phi(U z)=\phi(z)$ for any unitary transformation $U$ of $\mathbb{C}^{n} ; \phi$ is said to be separately radial if and only if $\phi(U z)=$ $\phi(z)$ for any unitary transformation $U$ of $\mathbb{C}^{n}$ with a diagonal matrix. This implies that a radial function has a form $\phi(z)=$ $\phi(|z|)$ and a separately radial function has a form $\phi(z)=$ $\phi\left(\left|z_{1}\right|, \ldots,\left|z_{n}\right|\right)$.

Using radial function and separately radial function, we give the following definition.

Definition 1. Let $p, s \in \mathbb{N}^{n}$ with $p \perp s$ and $f \in L^{1}\left(\mathbb{B}_{n}, d V\right)$.

(I) $f$ is called a quasihomogeneous function of degree $(p, s)$ if $f$ has the following decomposition:

$$
f(|r| \xi)=\xi^{p} \bar{\xi}^{s} \phi(|r|)
$$

for any $\xi \in \mathbb{S}_{n}, r=\left(r_{1}, r_{2}, \ldots, r_{n}\right)$ and $|r|=$ $\sqrt{\left|r_{1}\right|^{2}+\left|r_{2}\right|^{2}+\cdots+\left|r_{n}\right|^{2}}$, where $r_{i}=\left|z_{i}\right|$ for $1 \leq i \leq n$ and $\phi$ is a radial function. In this case, $T_{f}$ is called quasihomogeneous Toeplitz operator of degree $(p, s)$.

(II) $f$ is called a separately quasihomogeneous function of degree $(p, s)$ if $f$ has the following decomposition:

$$
f(|r| \xi)=\xi^{p} \bar{\xi}^{s} \phi(r)
$$

for any $\xi \in \mathbb{S}_{n}$, where $\phi$ is a separately radial function. In this case, $T_{f}$ is called separately quasihomogeneous Toeplitz operator of degree $(p, s)$.

We now recall some useful results from [34]. Denote by $\tau\left(\mathbb{B}_{n}\right)$ the base of $\mathbb{B}_{n}$, that is,

$$
\begin{gathered}
\tau\left(\mathbb{B}_{n}\right)=\left\{r=\left(r_{1}, r_{2}, \ldots, r_{n}\right)=\left(\left|z_{1}\right|,\left|z_{2}\right|, \ldots,\left|z_{n}\right|\right):\right. \\
\left.z=\left(z_{1}, z_{2}, \ldots, z_{n}\right) \in \mathbb{B}_{n}\right\} .
\end{gathered}
$$

If $\phi$ is a bounded separately radial function, we get that

$$
\int_{\mathbb{B}_{n}} \phi(z) d V(z)=2^{n} n ! \int_{\tau\left(\mathbb{B}_{n}\right)} \phi(r) r d r
$$

where $r d r=\prod_{i=1}^{n} r_{i} d r_{i}$.

Let $\mathscr{R}=\left\{\phi: \mathbb{B}_{n} \rightarrow \mathbb{C}\right.$ is separately radial : $\int_{\tau\left(\mathbb{B}_{n}\right)}|\phi(r)|^{2}$ $r d r<\infty\}$. Dong and Zhou [10] showed that for $f \in$ $L^{2}\left(\mathbb{B}_{n}, d V\right), f$ has the decomposition

$$
f(|r| \xi)=\sum_{p \perp s, p, s \in \mathbb{N}^{n}} \xi^{p} \bar{\xi}^{s} f_{p, s}(r), \quad f_{p, s} \in \mathscr{R} .
$$

They also proved the following result.
Lemma 2. Let $f(z)=\sum_{p \perp s, p, s \in \mathbb{N}^{n}} \xi^{p} \bar{\xi}^{s} f_{p, s}(r) \in L^{\infty}\left(\mathbb{B}_{n}, d V\right)$; then $\xi^{p} \bar{\xi}^{s} f_{p, s}(r)$ is bounded on $\mathbb{B}_{n}$ for multi-indexes $p, s \in \mathbb{N}^{n}$ with $p \perp s$.

In order to get our main results, we need to introduce the Mellin transform, which is defined for any function $\phi \in$ $L^{1}([0,1], r d r)$ by the formula

$$
\widehat{\phi}(z)=\int_{0}^{1} \phi(r) r^{z-1} d r .
$$

It is well known that $\widehat{\phi}$ is well defined on the right half-plane $\{z: \operatorname{Re} z \geq 2\}$ and is analytic on $\{z: \operatorname{Re} z>2\}$. It is helpful that the Mellin transform is uniquely determined by its value on an arithmetic sequence of integers. In fact, we have the following result (see [35, page 102]).

Lemma 3. Suppose that $\phi$ is a bounded analytic function on $\{z: \operatorname{Re} z>0\}$ which vanishes at the pairwise distinct points $z_{1}, z_{2}, \ldots$, where

$$
\begin{aligned}
& \text { (I) } \inf \left\{\left|z_{n}\right|\right\}>0 \text {, and } \\
& \text { (II) } \sum_{n \geq 1} \operatorname{Re}\left(1 / z_{n}\right)=\infty \text {. }
\end{aligned}
$$

Then $\phi$ vanishes identically on $\{z: \operatorname{Re} z>0\}$.

Remark 4. We will often use Lemma 3 to show that if $\phi \epsilon$ $L^{1}([0,1], r d r)$ and if there exists a sequence $\left\{n_{k}\right\} \subset \mathbb{N}$ such that

$$
\widehat{\phi}\left(n_{k}\right)=0, \quad \sum_{k \geq 0} \frac{1}{n_{k}}=\infty
$$

then $\widehat{\phi}(z)=0$ for all $z \in\{z: \operatorname{Re} z>2\}$ and $\operatorname{so} \phi=0$.

In this paper, we will need a similar result in higher dimensions. Now we give the following definition.

Definition 5. Let $E$ be a subset of $\mathbb{Z}_{+}^{2}$; one says that $E$ satisfies condition (I) if the following statement holds:

(I) there exists a sequence $\left\{\alpha_{i}^{(1)}\right\}_{i=1}^{\infty}$ such that $\sum_{i=1}^{\infty}=1 / \alpha_{i}^{(1)}$ $=\infty$, and for every fixed $\alpha_{i}^{(1)}$, there also exists a sequence $\left\{\alpha_{j(i)}^{(2)}\right\}_{j(i)=1}^{\infty}$ such that $\sum_{j(i)=1}^{\infty} 1 / \alpha_{j(i)}^{(2)}=\infty$ and $\left\{\left(\alpha_{i}^{(1)}, \alpha_{j(i)}^{(2)}\right)\right.$ : $j(i)=1,2, \ldots\} \subset E$.

Remark 6. It follows from Definition 5 that for a multi-index $\delta \in \mathbb{N}^{2}$, if $E$ is a subset of $\left\{\alpha \in \mathbb{Z}_{+}^{2}: \alpha \succeq \delta\right\}$ and if $E^{c}$ is the complement of $E$ in $\left\{\alpha \in \mathbb{Z}_{+}^{2}: \alpha \succeq \delta\right\}$, then either $E$ or $E^{c}$ satisfies condition (I).

In this paper, we will often use Lemmas 4 and 12 in [12] and Lemma 2.1 and Corollary 2.7 in [10] which can be stated as follows.

Lemma 7. Let $\phi$ be an integrable radial function on $\mathbb{B}_{n}$ such that $T_{\phi}$ is a bounded operator then for any multi-index $\alpha$,

$$
\begin{aligned}
& T_{\phi}\left(z^{\alpha}\right)=2(n+|\alpha|) ! \widehat{\phi}(2 n+2|\alpha|) z^{\alpha}, \\
& T_{\phi}\left(\bar{z}^{\alpha}\right)=2(n+|\alpha|) ! \widehat{\phi}(2 n+2|\alpha|) \bar{z}^{\alpha} .
\end{aligned}
$$


Lemma 8. Let $p$, s be two multi-indexes and let $\phi$ be an integrable radial function on $\mathbb{B}_{n}$ such that $T_{\xi^{p} \phi}, T_{\bar{\xi}^{s} \phi}$ and $T_{\xi^{p} \bar{\xi}^{s} \phi}$ are bounded operators. Then for any multi-index $\alpha$,

$$
\begin{aligned}
& T_{\xi\urcorner \bar{\xi}_{\phi}^{s}}\left(z^{\alpha}\right) \\
& =\left\{\begin{array}{cl}
\frac{2(p+\alpha) !(n+|p|+|\alpha|-|s|) !}{(p+\alpha-s) !(n-1+|\alpha|+|p|) !} \widehat{\phi} & \\
\times(2 n+2|\alpha|+|p|-|s|) z^{p+\alpha-s} & p+\alpha \geq s, \\
\frac{2 s !(n+|s|-|\alpha|-|p|) !}{(s-\alpha-p) !(n-1+|s|) !} & \\
\times(2 n+|s|-|p|) \bar{z}^{s-\alpha-p} & s \geq p+\alpha, \\
0 & s \neq p+\alpha, \\
& s \nsucceq p+\alpha, \\
& p+\alpha \nsucceq s ;
\end{array}\right.
\end{aligned}
$$$$
T_{\xi^{p} \bar{\xi}_{\phi}^{s}}\left(\bar{z}^{\alpha}\right)
$$$$
= \begin{cases}\frac{2(s+\alpha) !(n+|s|+|\alpha|-|p|) !}{(s+\alpha-p) !(n-1+|\alpha|+|s|) !} \widehat{\phi} & \\ \quad \times(2 n+2|\alpha|+|s|-|p|) \bar{z}^{s+\alpha-p} & s+\alpha \geq p, \\ \frac{2 p !(n+|p|-|\alpha|-|s|) !}{(p-\alpha-s) !(n-1+|p|) !} \widehat{\phi} & \\ \times(2 n+|p|-|s|) z^{p-\alpha-s} & p \geq s+\alpha, \\ & p \neq s+\alpha, \\ 0 & p \nsucceq s+\alpha, \\ & s+\alpha \neq p .\end{cases}
$$

In particular, if $p$, s are two nonzero multi-indexes with $p \perp s$, one has

$$
\begin{aligned}
& T_{\xi_{p} \bar{\xi}_{\phi}^{s}}\left(z^{\alpha}\right) \\
& =\left\{\begin{array}{cc}
\frac{2(p+\alpha) !(n+|p|+|\alpha|-|s|) !}{(p+\alpha-s) !(n-1+|\alpha|+|p|) !} \widehat{\phi} & \\
\quad \times(2 n+2|\alpha|+|p|-|s|) z^{p+\alpha-s} & \alpha \succeq s, \\
0 & \alpha \nsucceq s ;
\end{array}\right. \\
& T_{\xi_{p} \bar{\xi}_{\phi}^{s}\left(\bar{z}^{\alpha}\right)} \\
& =\left\{\begin{array}{cc}
\frac{2(s+\alpha) !(n+|s|+|\alpha|-|p|) !}{(s+\alpha-p) !(n-1+|\alpha|+|s|) !} \widehat{\phi} & \\
\times(2 n+2|\alpha|+|s|-|p|) \bar{z}^{s+\alpha-p} & \alpha \succeq p, \\
0 & \alpha \nsucceq p .
\end{array}\right.
\end{aligned}
$$

Lemma 9. Let $\alpha, \beta \in \mathbb{N}^{n}$ with $\alpha \neq \beta$ and let $t \in[0,1)$. If $\phi$ is a bounded separately radial function on $\mathbb{B}_{n}$, then

$$
\int_{\mathbb{S}_{n}} \phi(t \xi) \xi^{\alpha} \bar{\xi}^{\beta} d \sigma(\xi)=0 .
$$

Lemma 10. Let $p, s \in \mathbb{N}^{2}$ and let $g(r)$ be a bounded function on $\tau\left(\mathbb{B}_{2}\right)$. If the set

$$
E=\left\{\alpha \in \mathbb{Z}_{+}^{2}: \alpha \geq s, \int_{\tau\left(\mathbb{B}_{2}\right)} g(r) r^{2 \alpha+p-s} r d r=0\right\}
$$

satisfies condition (I), then $g=0$.

\section{The Product of Toeplitz Operators with Separately Quasihomogeneous Symbols}

We start this section with the following result.

Lemma 11. Let $\phi$ be a bounded separately radial function on $\mathbb{B}_{n}$; then for any multi-index $\alpha \in \mathbb{N}^{n}$,

$$
\begin{aligned}
& T_{\phi}\left(z^{\alpha}\right)=\frac{2^{n}(n+|\alpha|) ! \int_{\tau\left(\mathbb{B}_{n}\right)} \phi(r) r^{2 \alpha} r d r}{\alpha !} z^{\alpha}, \\
& T_{\phi}\left(\bar{z}^{\alpha}\right)=\frac{2^{n}(n+|\alpha|) ! \int_{\tau\left(\mathbb{B}_{n}\right)} \phi(r) r^{2 \alpha} r d r}{\alpha !} \bar{z}^{\alpha} .
\end{aligned}
$$

Proof. For multi-indexes $\alpha, \beta \in \mathbb{N}^{n}$, it is well known that

$$
\left\langle z^{\alpha}, z^{\beta}\right\rangle= \begin{cases}0 & \alpha \neq \beta, \\ \frac{n ! \alpha !}{(n+|\alpha|) !} & \alpha=\beta .\end{cases}
$$

According to (9) and Lemma 9, we get that

$$
\begin{aligned}
\left\langle T_{\phi} z^{\alpha}, z^{\beta}\right\rangle & =\left\langle\phi z^{\alpha}, z^{\beta}\right\rangle \\
& =\int_{\mathbb{B}_{n}} \phi(z) z^{\alpha} \bar{z}^{\beta} d V(z) \\
& = \begin{cases}0 & \alpha \neq \beta, \\
2^{n} n ! \int_{\mathcal{\tau}\left(\mathbb{B}_{n}\right)} \phi(r) r^{2 \alpha} r d r & \alpha=\beta .\end{cases}
\end{aligned}
$$

Similarly, if $\beta \succeq 0, \beta \neq 0$, we obtain $\left\langle T_{\phi} z^{\alpha}, \bar{z}^{\beta}\right\rangle=0=\left\langle z^{\alpha}, \bar{z}^{\beta}\right\rangle$. Since $\left\{z^{\beta}\right\}_{\beta \succeq 0} \cup\left\{\bar{z}^{\beta}\right\}_{\beta \succeq 0, \beta \neq 0}$ is a basis of the pluriharmonic Bergman space, we have

$$
T_{\phi}\left(z^{\alpha}\right)=\frac{2^{n}(n+|\alpha|) ! \int_{\tau\left(\mathbb{B}_{n}\right)} \phi(r) r^{2 \alpha} r d r}{\alpha !} z^{\alpha} .
$$

By a similar argument, one can deduce that $T_{\phi}\left(\bar{z}^{\alpha}\right)=\left(\left(2^{n}(n+\right.\right.$ $\left.\left.|\alpha|) ! \int_{\tau\left(\mathbb{B}_{n}\right)} \phi(r) r^{2 \alpha} r d r\right) / \alpha !\right) \bar{z}^{\alpha}$. This completes the proof.

The following theorem is crucial for us to get our main results.

Theorem 12. Let $f$ be a bounded function on $\mathbb{D}$. Then the following conditions are equivalent:

(a) for any $\alpha \in \mathbb{N}^{n}$, there exists $\lambda_{\alpha} \in \mathbb{C}$ such that $T_{f}\left(z^{\alpha}\right)=$ $\lambda_{\alpha} z^{\alpha}$

(b) for any $\alpha \in \mathbb{N}^{n}$, there exists $\lambda_{\alpha} \in \mathbb{C}$ such that $T_{f}\left(\bar{z}^{\alpha}\right)=$ $\lambda_{\alpha} \bar{z}^{\alpha}$

Proof. Assume (a) holds; that is, $T_{f}\left(z^{\alpha}\right)=\lambda_{\alpha} z^{\alpha}$. For any multi-index $\beta \in \mathbb{N}^{n}$, it follows from (19) that

$$
\begin{aligned}
\left\langle T_{f} \bar{z}^{\alpha}, \bar{z}^{\beta}\right\rangle & =\left\langle f \bar{z}^{\alpha}, \bar{z}^{\beta}\right\rangle=\left\langle f z^{\beta}, z^{\alpha}\right\rangle \\
& =\left\langle T_{f} z^{\beta}, z^{\alpha}\right\rangle=\lambda_{\beta}\left\langle z^{\beta}, z^{\alpha}\right\rangle \\
& = \begin{cases}0 & \alpha \neq \beta, \\
\lambda_{\alpha}\left\langle z^{\alpha}, z^{\alpha}\right\rangle & \alpha=\beta\end{cases}
\end{aligned}
$$




$$
\begin{aligned}
& =\lambda_{\alpha}\left\langle\bar{z}^{\alpha}, \bar{z}^{\beta}\right\rangle, \\
\left\langle T_{f} \bar{z}^{\alpha}, z^{\beta}\right\rangle & =\left\langle f \bar{z}^{\alpha}, z^{\beta}\right\rangle=\left\langle f z^{0}, z^{\alpha+\beta}\right\rangle \\
& =\left\langle T_{f} z^{0}, z^{\alpha+\beta}\right\rangle=\lambda_{0}\left\langle z^{0}, z^{\alpha+\beta}\right\rangle \\
& = \begin{cases}0 & \alpha \neq 0 \text { or } \beta \neq 0, \\
\lambda_{0} & \alpha=\beta=0\end{cases} \\
& =\lambda_{\alpha}\left\langle\bar{z}^{\alpha}, z^{\beta}\right\rangle .
\end{aligned}
$$

$\left\{z^{\beta}\right\}_{\beta \succeq 0} \cup\left\{\bar{z}^{\beta}\right\}_{\beta \succeq 0}$ which is a basis of the pluriharmonic Bergman space implies that $T_{f}\left(\bar{z}^{\alpha}\right)=\lambda_{\alpha} \bar{z}^{\alpha}$.

By a similar argument, one can show that (b) implies (a), which completes the proof.

Using Theorem 12, we give the necessary and sufficient condition when a bounded function is a separately radial function.

Theorem 13. Let $\phi \in L^{\infty}\left(\mathbb{B}_{n}, d V\right)$. Then the following statements are equivalent:

(a) for any $\alpha \in \mathbb{N}^{n}$, there exists $\lambda_{\alpha} \in \mathbb{C}$ such that $T_{\phi}\left(z^{\alpha}\right)=$ $\lambda_{\alpha} z^{\alpha}$

(b) $\phi$ is a separately radial function.

Proof. It is easy to show that (b) implies (a) by Lemma 11.

Now suppose (a) holds. That is, $T_{\phi}\left(z^{\alpha}\right)=\lambda_{\alpha} z^{\alpha}$. Then for any unitary transformation $U$ of $\mathbb{C}^{n}$ with diagonal matrix and $z \in \mathbb{B}_{n}$, we get $U^{-1} z \in \mathbb{B}_{n}$. Hence, one can obtain $T_{\phi}\left(U^{-1} z\right)^{\alpha}=\lambda_{\alpha}\left(U^{-1} z\right)^{\alpha}$. A direct calculation shows that

$$
\begin{aligned}
P\left[\phi(U z) z^{\alpha}\right](w) & =\int_{\mathbb{B}_{n}} \frac{\phi(U z) z^{\alpha}}{(1-\langle w, z\rangle)^{n+1}} d V(z) \\
& =\int_{\mathbb{B}_{n}} \frac{\phi(z)\left(U^{-1} z\right)^{\alpha}}{\left(1-\left\langle w, U^{-1} z\right\rangle\right)^{n+1}} d V(z) \\
& =\int_{\mathbb{B}_{n}} \frac{\phi(z)\left(U^{-1} z\right)^{\alpha}}{(1-\langle U w, z\rangle)^{n+1}} d V(z) \\
& =P\left[\phi(z)\left(U^{-1} z\right)^{\alpha}\right](U w) .
\end{aligned}
$$

Similarly, we get that

$$
\begin{gathered}
P\left[\overline{\phi(U z) z^{\alpha}}\right](w)=P\left[\overline{\phi(z)\left(U^{-1} z\right)^{\alpha}}\right](U w), \\
P\left[\phi(U z) z^{\alpha}\right](0)=P\left[\phi(z)\left(U^{-1} z\right)^{\alpha}\right](0) .
\end{gathered}
$$

It follows that

$$
\begin{aligned}
T_{\phi \circ U}\left(z^{\alpha}\right)(w)= & P\left[\phi(U z) z^{\alpha}\right](w) \\
& +\overline{P\left[\overline{\phi(U z) z^{\alpha}}\right]}(w)-P\left[\phi(U z) z^{\alpha}\right](0)
\end{aligned}
$$

$$
\begin{aligned}
= & P\left[\phi(z)\left(U^{-1} z\right)^{\alpha}\right](U w)+\overline{P\left[\overline{\phi(z)\left(U^{-1} z\right)^{\alpha}}\right]} \\
& \times(U w)-P\left[\phi(z)\left(U^{-1} z\right)^{\alpha}\right](0) \\
= & T_{\phi}\left(\left(U^{-1} z\right)^{\alpha}\right)(U w)=\lambda_{\alpha}\left(U^{-1} z\right)^{\alpha}(U w) \\
= & \lambda_{\alpha} w^{\alpha}=T_{\phi}\left(z^{\alpha}\right)(w) .
\end{aligned}
$$

By Theorem 12, we have $T_{\phi \circ U}\left(\bar{z}^{\alpha}\right)=\lambda_{\alpha} \bar{z}^{\alpha}=T_{\phi}\left(\bar{z}^{\alpha}\right)$. Consequently, one can get that $T_{\phi \circ U}=T_{\phi}$ and so $\phi \circ U=\phi$. Then $\phi$ is a separately radial function. This completes the proof.

A direct calculation gives the following lemma, which we will use often.

Lemma 14. Let $p, s$ be two multi-indexes and let $\phi \in \mathscr{R}$ and $\xi^{p} \bar{\xi}^{s} \phi \in L^{\infty}\left(\mathbb{B}_{n}, d V\right)$. Then for any multi-index $\alpha \in \mathbb{N}^{n}$,

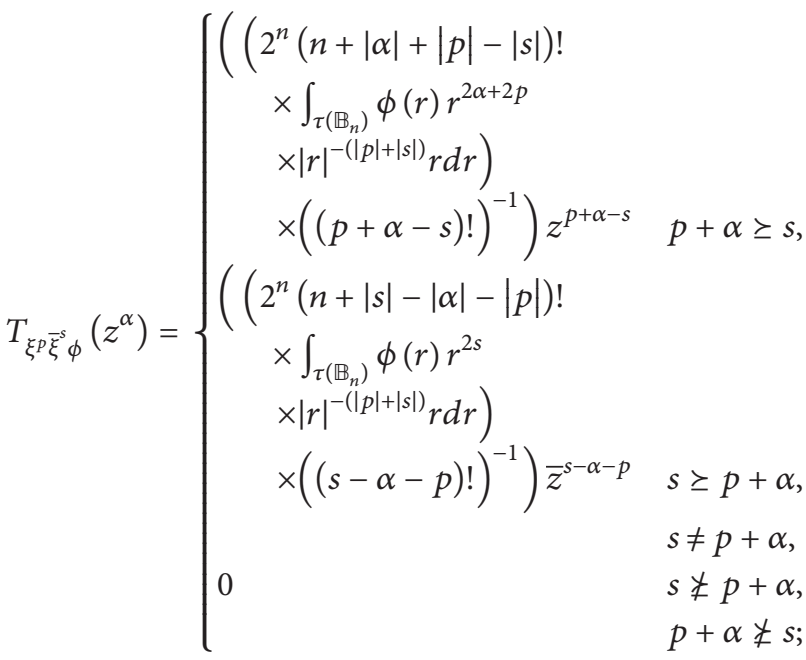

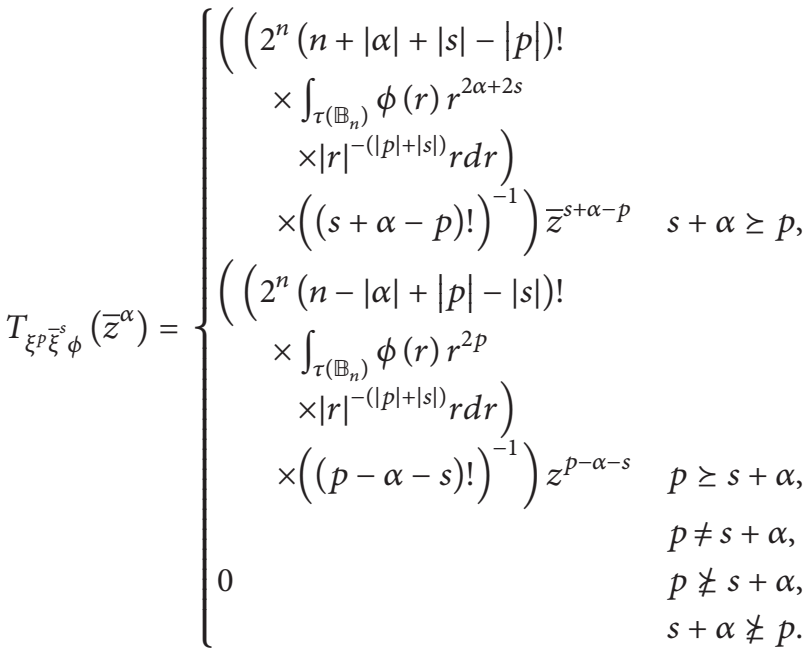


Furthermore, if $p, s$ are two nonzero multi-indexes with $p \perp s$, one has

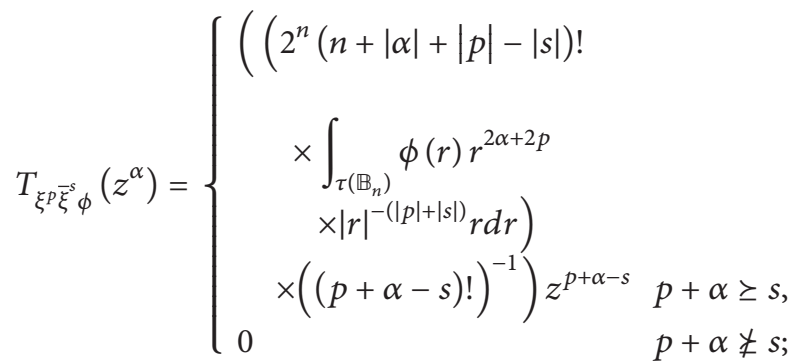

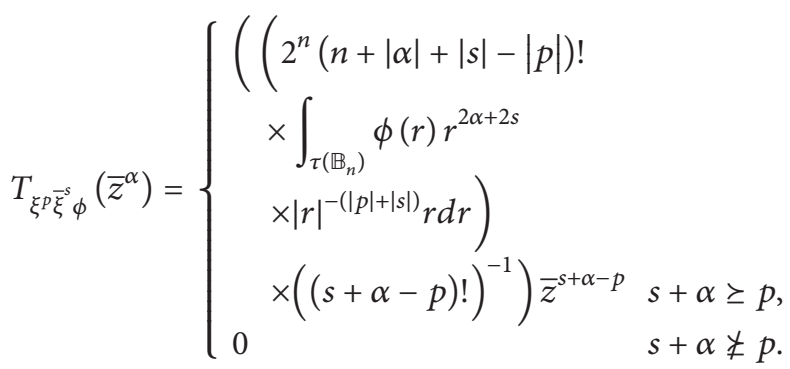

Proof. Here, we only prove that (26) and (27) hold. For any multi-index $\beta \in \mathbb{N}^{n}$, if $p+\alpha \nsucceq s$, then there exists $i$ such that $\alpha_{i}+p_{i}<s_{i}$. Hence, $p+\alpha \neq \beta+s$. It follows from Lemma 9 that

$$
\begin{aligned}
\left\langle P\left[\xi^{p} \bar{\xi}^{s} \phi z^{\alpha}\right], z^{\beta}\right\rangle= & \int_{\mathbb{B}_{n}} \xi^{p} \bar{\xi}^{s} \phi(z) z^{\alpha} \bar{z}^{\beta} d V(z) \\
= & \int_{0}^{1} 2 n|r|^{2 n-1}|r|^{|\alpha|+|\beta|} d|r| \\
& \times \int_{\mathbb{S}_{n}} \phi(|r| \xi) \xi^{p+\alpha} \bar{\xi}^{s+\beta} d \sigma(\xi)=0 .
\end{aligned}
$$

For $\alpha+p \succeq s$, using Lemma 9, (9), and (19), we get

$$
\begin{aligned}
& \left\langle P\left[\xi^{p} \bar{\xi}^{s} \phi z^{\alpha}\right], z^{\beta}\right\rangle \\
& =\int_{\mathbb{B}_{n}} \xi^{p} \bar{\xi}^{s} \phi(z) z^{\alpha} \bar{z}^{\beta} d V(z) \\
& = \begin{cases}0 & \\
2^{n} n ! \int_{\tau\left(\mathbb{B}_{n}\right)} \phi(r) r^{2 \alpha+2 p} & \\
\times|r|^{-(|p|+|s|)} r d r & \beta \neq p+\alpha-s,\end{cases} \\
& \left\langle z^{p+\alpha-s}, z^{\beta}\right\rangle=\left\{\begin{array}{cc}
0 & \beta \neq \alpha-s, \\
\frac{n !(p+\alpha-s) !}{(n+|\alpha|+|p|-|s|) !} & \beta=p+\alpha-s .
\end{array}\right.
\end{aligned}
$$

Moreover, for $\beta \succeq 0, \beta \neq 0$, we have $\left\langle P\left[\xi^{p} \bar{\xi}^{s} \phi z^{\alpha}\right], \bar{z}^{\beta}\right\rangle=0$. It follows that for $p+\alpha \geq s,\left\langle P\left[\xi^{p} \bar{\xi}^{s} \phi z^{\alpha}\right], \bar{z}^{\beta}\right\rangle=\left\langle z^{p+\alpha-s}, \bar{z}^{\beta}\right\rangle=0$. Thus, one can obtain that

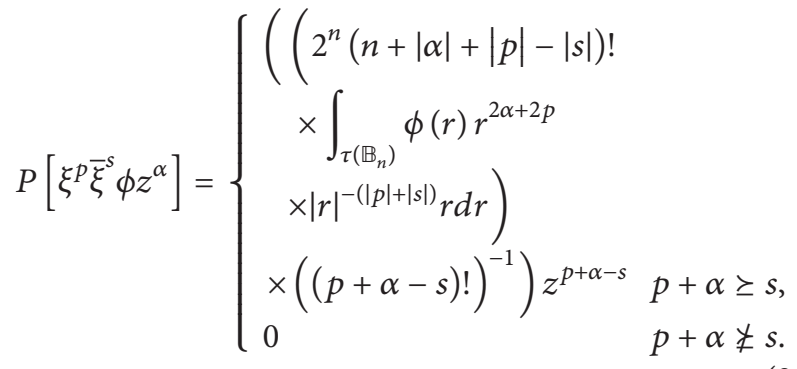

Note that $\bar{\phi}$ is still a separately radial function; by a similar argument, we get that

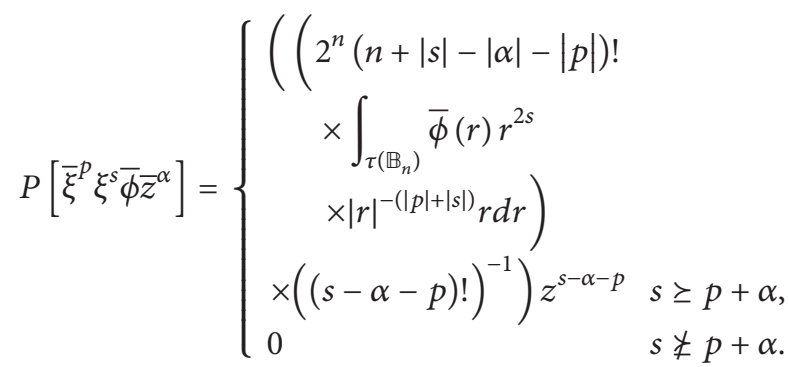

It follows from above two equations that

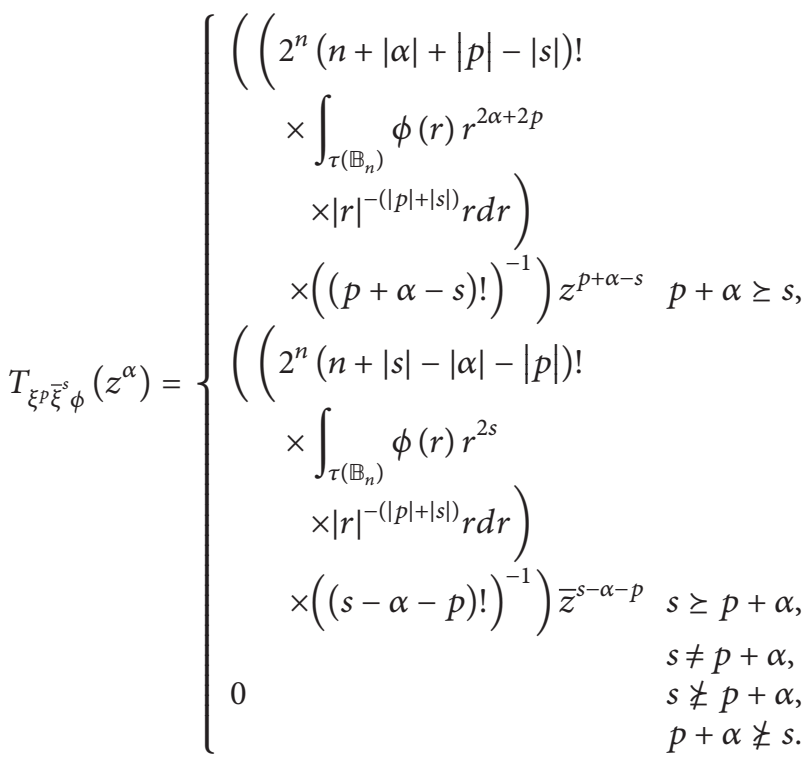

Furthermore, if $p \perp s$ and $p, s$ are nonzero multi-indexes, there exists $i \in\{1,2, \ldots, n\}$ such that $p_{i}>0$ and $s_{i}=0$. This implies that there does not exist $\alpha \in \mathbb{N}^{n}$ such that $s \geq p+\alpha$. It follows that (27) holds. This completes the proof.

Theorem 15. Let $p, s \in \mathbb{N}^{n}$ be two nonzero multi-indexes with $p \perp s$ and let $\phi$ be a bounded function on $\mathbb{B}_{n}$. Then the following conditions are equivalent:

(a) for any $\alpha \in \mathbb{N}^{n}$, there exists $\lambda_{\alpha} \in \mathbb{C}$ such that

$$
T_{\phi}\left(z^{\alpha}\right)= \begin{cases}0 & p+\alpha \nsucceq s, \\ \lambda_{\alpha} z^{p+\alpha-s} & p+\alpha \succeq s ;\end{cases}
$$


(b) $\phi$ is a separately quasihomogeneous function of degree $(p, s)$.

Proof. It is obvious that (b) implies (a). Now assume (a) holds. For any multi-index $\beta \in \mathbb{N}^{n}$, we have

$$
\begin{aligned}
\left\langle T_{\bar{z}^{p} z^{s} \phi}\left(z^{\alpha}\right), z^{\beta}\right\rangle & =\left\langle\phi z^{\alpha+s}, z^{p+\beta}\right\rangle=\left\langle T_{\phi} z^{\alpha+s}, z^{p+\beta}\right\rangle \\
& =\left\langle\lambda_{\alpha+s} z^{\alpha+p}, z^{p+\beta}\right\rangle \\
& = \begin{cases}0 & \alpha \neq \beta, \\
\frac{\lambda_{\alpha+s} n !(\alpha+p) !}{(n+|\alpha|+|p|) !} & \alpha=\beta .\end{cases}
\end{aligned}
$$

Similarly, for $\beta \geq 0$ and $\beta \neq 0$, we get that $\left\langle T_{\bar{z}^{p} z^{s} \phi}\left(z^{\alpha}\right), \bar{z}^{\beta}\right\rangle=$ $0=\left\langle z^{\alpha}, \bar{z}^{\beta}\right\rangle$. In light of (19), one can deduce that

$$
T_{\bar{z}^{p} z^{s} \phi}\left(z^{\alpha}\right)=\frac{\lambda_{\alpha+s}(\alpha+p) !(n+|\alpha|) !}{\alpha !(n+|\alpha|+|p|) !} z^{\alpha} .
$$

It follows from Theorem 13 that $\bar{z}^{p} z^{s} \phi$ is a separately radial function. Let $\bar{z}^{p} z^{s} \phi(z)=\psi(z)$, where $\psi$ is a separately radial function on $\mathbb{B}_{n}$. It follows that

$$
\phi(z)=\psi(r) r^{-(2 p+2 s)}|r|^{(|p|+|s|)} \xi^{p} \bar{\xi}^{s},
$$

which implies that $\phi$ is a separately quasihomogeneous function of degree $(p, s)$.

Now we discuss when the product of two Toeplitz operators with certain symbols is a Toeplitz operator.

Theorem 16. Let $\phi_{1}, \phi_{2}$ be two bounded separately radial functions on $\mathbb{B}_{n}$. If there exists a bounded function $h$ such that $T_{\phi_{1}} T_{\phi_{2}}=T_{h}$, then $h$ is also a separately radial function on $\mathbb{B}_{n}$.

Proof. It follows from Lemma 11 that for $\alpha \in \mathbb{N}^{n}$

$$
\begin{aligned}
T_{h}\left(z^{\alpha}\right)= & T_{\phi_{1}} T_{\phi_{2}}\left(z^{\alpha}\right) \\
= & \frac{2^{n}(n+|\alpha|) ! \int_{\tau\left(\mathbb{B}_{n}\right)} \phi_{2}(r) r^{2 \alpha} r d r}{\alpha !} \\
& \cdot \frac{2^{n}(n+|\alpha|) ! \int_{\tau\left(\mathbb{B}_{n}\right)} \phi_{1}(r) r^{2 \alpha} r d r}{\alpha !} z^{\alpha} .
\end{aligned}
$$

In virtue of Theorem 13, we get that $h$ is a separately radial function. This completes the proof.

Theorem 17. Let $p, s \in \mathbb{N}^{n}$ be two nonzero multi-indexes with $p \perp s$ and let $\phi_{1}, \phi_{2} \in \mathscr{R}$ such that $\bar{\xi}^{s} \phi_{1}, \xi^{p} \phi_{2} \in L^{\infty}\left(\mathbb{B}_{n}, d V\right)$. If there exists a bounded function $h$ such that $T_{\vec{\xi}^{s} \phi_{1}} T_{\xi^{p} \phi_{2}}=T_{h}$, then $h$ is a separately quasihomogeneous function of degree $(p, s)$.

Proof. For any multi-index $\alpha \in \mathbb{N}^{n}$, using Lemma 14 , one can obtain that

$$
T_{h}\left(z^{\alpha}\right)=T_{\bar{\xi}^{s} \phi_{1}} T_{\xi^{p} \phi_{2}}\left(z^{\alpha}\right)= \begin{cases}\lambda_{\alpha} z^{\alpha+p-s} & \alpha+p \succeq s, \\ 0 & \alpha+p \nsucceq s,\end{cases}
$$

where $\lambda_{\alpha}=\left(2^{n}(n+|\alpha|+|p|-|s|) ! \int_{\tau\left(\mathbb{B}_{n}\right)}\right.$ $\left.\phi_{1}(r) r^{2 \alpha+2 p}|r|^{-|s|} r d r /(p+\alpha-s) !\right) \cdot\left(2^{n}(n+|\alpha|+\right.$ $\left.|p|) ! \int_{\mathcal{\tau}\left(\mathbb{B}_{n}\right)} \phi_{2}(r) r^{2 \alpha+2 p}|r|^{-|p|} r d r /(p+\alpha) !\right)$. It follows from Theorem 15 that $h$ is a separately quasihomogeneous function of degree $(p, s)$.

Using the same technique, we give the following result and omit the proof.

Theorem 18. Let $p, s \in \mathbb{N}^{n}$ be two nonzero multi-indexes with $p \perp s$ and let $\phi_{1}, \phi_{2} \in \mathscr{R}$ such that $\phi_{1}, \xi^{p} \bar{\xi}^{s} \phi_{2} \in L^{\infty}\left(\mathbb{B}_{n}, d V\right)$. If there exists a bounded function $h$ such that $T_{\phi_{1}} T_{\xi^{p} \bar{\xi}^{s} \phi_{2}}=T_{h}$, then $h$ is a separately quasihomogeneous function of degree $(p, s)$.

\section{The Zero-Product Problem of Toeplitz Operators with Quasihomogeneous and Separately Quasihomogeneous Symbols}

In this section, we will study the zero-product problem for several Toeplitz operators when one of the symbols is separately quasihomogeneous and the others are homogeneous, and show that the zero-product problem for two Toeplitz operators with certain symbols has only a trivial solution on the pluriharmonic Bergman space of the unit ball.

Theorem 19. Let $p_{i}, s_{i}(1 \leq i \leq m) \in \mathbb{N}^{n}$ be nonzero multi-indexes with $p_{i} \perp s_{i}$ and let $\phi_{1}, \phi_{2}, \ldots, \phi_{m-1}$ be square integrable radial functions and $\phi_{m} \in \mathscr{R}$ such that $\xi^{p_{i}} \bar{\xi}^{s_{i}} \phi_{i} \in$ $L^{\infty}\left(\mathbb{B}_{n}, d V\right)$. Then

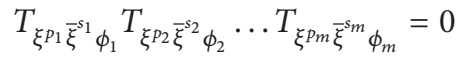

if and only if $\phi_{i}=0$ for some $i$.

Proof. Suppose $T_{\xi^{p_{1}} \bar{\xi}^{s_{1}} \phi_{1}} T_{\xi^{p_{2}} \bar{\xi}^{s_{2}} \phi_{2}} \ldots T_{\xi^{p_{m}} \bar{\xi}^{s_{m}} \phi_{m}}=0$. Then for multi-index $\alpha \in \mathbb{N}^{n}$,

$$
\begin{aligned}
& T_{\xi^{p_{1}} \bar{\xi}^{s_{1}} \phi_{1}} T_{\xi^{p_{2}} \bar{\xi}^{s_{2}^{2}} \phi_{2}} \ldots T_{\xi^{p_{m}} \bar{\xi}^{s_{m}} \phi_{m}}\left(z^{\alpha}\right)=0, \\
& T_{\xi^{p_{1}} \bar{\xi}^{s_{1}} \phi_{1}} T_{\xi^{p_{2}} \bar{\xi}^{s^{2}} \phi_{2}} \ldots T_{\xi^{p_{m}} \bar{\xi}^{s_{m}} \phi_{m}}\left(\bar{z}^{\alpha}\right)=0 .
\end{aligned}
$$

It follows from Lemmas 8 and 14 that

$$
\begin{aligned}
& T_{\xi^{p} \vec{\xi}^{s_{1}} \phi_{1}} T_{\xi^{p_{2}} \bar{\xi}^{\xi^{2}} \phi_{2}} \ldots T_{\xi^{p m} \vec{\xi}^{s} \phi_{m}}\left(z^{\alpha}\right) \\
& =\left\{\begin{aligned}
C_{\alpha} z^{\alpha+\sum_{j=0}^{m-1} p_{m-j}-\sum_{j=0}^{m-1} s_{m-j}} & \\
\alpha+p_{m} \geq s_{m}, \ldots, \alpha+\sum_{j=0}^{m-1} p_{m-j} & \geq \sum_{j=0}^{m-1} s_{m-j}, \\
0 & \text { others, }
\end{aligned}\right.
\end{aligned}
$$


where

$$
\begin{aligned}
& C_{\alpha}=\left(2^{n}\left(n+|\alpha|+\left|p_{m}\right|-\left|s_{m}\right|\right) !\right. \\
& \left.\times \int_{\tau\left(\mathbb{B}_{n}\right)} \phi_{m}(r) r^{2 \alpha+2 p_{m}}|r|^{-\left(\left|p_{m}\right|+\left|s_{m}\right|\right)} r d r\right) \\
& \times\left(\left(p_{m}+\alpha-s_{m}\right) !\right)^{-1} \\
& \cdot \prod_{i=1}^{m-1}\left\{\left(2\left(\alpha+\sum_{j=0}^{i} p_{m-j}-\sum_{j=0}^{i-1} s_{m-j}\right) !\right.\right. \\
& \left.\times\left(n+|\alpha|+\sum_{j=0}^{i}\left|p_{m-j}\right|-\sum_{j=0}^{i}\left|s_{m-j}\right|\right) !\right) \\
& \times\left(\left(\alpha+\sum_{j=0}^{i} p_{m-j}-\sum_{j=0}^{i} s_{m-j}\right) !\right. \\
& \left.\times\left(n-1+|\alpha|+\sum_{j=0}^{i}\left|p_{m-j}\right|-\sum_{j=0}^{i-1}\left|s_{m-j}\right|\right) !\right)^{-1} \\
& \times \widehat{\phi_{m-i}}\left(2 n+2|\alpha|+2 \sum_{j=0}^{i-1}\left|p_{m-j}\right|\right. \\
& \left.\left.-2 \sum_{j=0}^{i-1}\left|s_{m-j}\right|+\left|p_{m-i}\right|-\left|s_{m-i}\right|\right)\right\} .
\end{aligned}
$$

If $T_{\xi^{p_{1}} \bar{\xi}^{s_{1}} \phi_{1}} T_{\xi^{p_{2}} \bar{\xi}^{s_{2}} \phi_{2}} \ldots T_{\xi^{p_{m}} \bar{\xi}^{s_{m}} \phi_{m}}\left(z^{\alpha}\right)=0$, then, for any multiindex $\alpha \in \mathbb{N}^{n}$ such that $\alpha+p_{m} \geq s_{m}, \ldots, \alpha+\sum_{j=0}^{m-1} p_{m-j} \succeq$ $\sum_{j=0}^{m-1} s_{m-j}$, we have

$$
\begin{gathered}
\int_{\tau\left(\mathbb{B}_{n}\right)} \phi_{m}(r) r^{2 \alpha+2 p_{m}}|r|^{-\left(\left|p_{m}\right|+\left|s_{m}\right|\right)} r d r \\
\cdot \prod_{i=1}^{m-1} \widehat{\phi_{m-i}}\left(2 n+2|\alpha|+2 \sum_{j=0}^{i-1}\left|p_{m-j}\right|-2 \sum_{j=0}^{i-1}\left|s_{m-j}\right|\right. \\
\left.+\left|p_{m-i}\right|-\left|s_{m-i}\right|\right)=0 .
\end{gathered}
$$

Now we are ready to show that $\phi_{i}=0$ for some $i$. For the sake of simplicity, we will consider the case of $n=2$. Let $E=\left\{\alpha \succeq \sum_{j=0}^{m-1} s_{m-j}: \int_{\tau\left(\mathbb{B}_{n}\right)} \phi_{m}(r) r^{2 \alpha+2 p_{m}}|r|^{-\left(\left|p_{m}\right|+\left|s_{m}\right|\right)} r d r=\right.$ $0\}$. If $E$ satisfies condition (I), then $\phi_{m}=0$ by Lemma 10 . Otherwise, let $E^{c}$ denote the complement of $E$ in $\left\{\alpha \in \mathbb{Z}_{+}^{2}\right.$ : $\left.\alpha \succeq \sum_{j=0}^{m-1} s_{m-j}\right\}$, and it follows from Remark 6 that $E^{c}$ satisfies condition (I). Now let

$$
\begin{gathered}
M=\left\{\alpha+p_{m} \geq s_{m}, \ldots, \alpha+\sum_{j=0}^{m-1} p_{m-j}\right. \\
\geq \sum_{j=0}^{m-1} s_{m-j}: \int_{\tau\left(\mathbb{B}_{n}\right)} \phi_{m}(r) r^{2 \alpha+2 p_{m}} \\
\left.\times|r|^{-\left(\left|p_{m}\right|+\left|s_{m}\right|\right)} r d r \neq 0\right\} .
\end{gathered}
$$

It is obvious that $M \supset E^{c}$, which implies that $M$ satisfies condition (I). Furthermore, we get that

$$
\sum_{\alpha \in M} \frac{1}{|\alpha|}=\infty \text {. }
$$

Set

$$
\begin{gathered}
M_{i}=\left\{\alpha+p_{m} \geq s_{m}, \ldots, \alpha+\sum_{j=0}^{m-1} p_{m-j} \geq \sum_{j=0}^{m-1} s_{m-j}:\right. \\
\widehat{\phi}_{i}\left(2 n+2|\alpha|+2 \sum_{j=0}^{m-i-1}\right. \\
\times\left|p_{m-j}\right|-2 \sum_{j=0}^{m-i-1}\left|s_{m-j}\right| \\
\left.+\left|p_{i}\right|-\left|s_{i}\right|\right)=0
\end{gathered}
$$

for $1 \leq i \leq m-1$. According to (43), we obtain that $M \subset$ $\cup_{i=1}^{m-1} M_{i}$. Hence there exists some $i$ such that

$$
\sum_{\alpha \in M_{i}} \frac{1}{|\alpha|}=\infty
$$

By Remark 4, one can deduce that $\phi_{i}=0$ for some $i \quad(1 \leq i \leq$ $m-1)$. Moreover, if $\phi_{i}=0$ for some $i(1 \leq i \leq m)$, then $T_{\xi^{p_{1}} \bar{\xi}^{s_{1}} \phi_{1}} T_{\xi^{p_{2}} \bar{\xi}^{s_{2}} \phi_{2}} \ldots T_{\xi^{p_{m}} \bar{\xi}^{s_{m}} \phi_{m}}\left(\bar{z}^{\alpha}\right)=0$.

Conversely, it is obvious that if $\phi_{i}=0$ for some $i$, then

$$
T_{\xi^{p_{1}} \bar{\xi}^{s_{1}} \phi_{1}} T_{\xi^{p_{2}} \bar{\xi}^{s_{2}} \phi_{2}} \cdots T_{\xi^{p_{m}} \bar{\xi}^{s_{m}} \phi_{m}}=0 .
$$

This completes the proof.

The following result is a partial answer to the zeroproduct problem for two Toeplitz operators on $L_{h}^{2}\left(\mathbb{B}_{n}\right)$.

Theorem 20. Let $f(z)=\sum_{p, s \in \mathbb{N}^{n}, p \neq 0, s \neq 0, p \perp s} \xi^{p} \bar{\xi}^{s} f_{p, s}(r) \in$ $L^{\infty}\left(\mathbb{B}_{n}, d V\right)$, where $f_{p, s}(r) \in \mathscr{R}$. Let $g=\xi^{p^{*}} \bar{\xi}^{s^{*}} \phi(r) \in$ $L^{\infty}\left(\mathbb{B}_{n}, d V\right)$, where $p^{*}, s^{*}$ are two nonzero indexes with $p^{*} \perp$ $s^{*}$ and $\phi \in \mathscr{R}$. Then $T_{f} T_{g}=0$ if and only if either $f=0$ or $g=0$. 
Proof. By Lemma 2, we get that $\xi^{p} \bar{\xi}^{s} f_{p, s}(r)$ is bounded for any multi-indexes $p, s \in \mathbb{N}^{n}, p \neq 0, s \neq 0, p \perp s$.

If $T_{f} T_{g}=0$, then for any multi-index $\alpha \in \mathbb{N}^{n}, T_{f} T_{g}\left(z^{\alpha}\right)=$ 0 and $T_{f} T_{g}\left(\bar{z}^{\alpha}\right)=0$. It follows from (27) that

$$
\begin{aligned}
& T_{\xi p \bar{\xi}^{s} f_{p, s}(r)}\left(z^{\alpha}\right) \\
& =c(p, s, \alpha) \int_{\tau\left(\mathbb{B}_{n}\right)} f_{p, s}(r) r^{2 \alpha+2 p} \\
& \quad \times|r|^{-(|p|+|s|)} r d r z^{\alpha+p-s},
\end{aligned}
$$

where

$$
c(p, s, \alpha)= \begin{cases}0 & p+\alpha \nsucceq s, \\ \frac{2^{n}(n+|\alpha|+|p|-|s|) !}{(p+\alpha-s) !} & p+\alpha \succeq s\end{cases}
$$

and consequently,

$$
\begin{aligned}
T_{f}\left(z^{\alpha}\right)= & \sum_{p, s \in \mathbb{N}^{n}, p \neq 0, s \neq 0, p \perp s} c(p, s, \alpha) \\
& \times \int_{\tau\left(\mathbb{B}_{n}\right)} f_{p, s}(r) r^{2 \alpha+2 p}|r|^{-(|p|+|s|)} r d r z^{\alpha+p-s} .
\end{aligned}
$$

$T_{f} T_{g}\left(z^{\alpha}\right)=0$ together with (27) implies that for any multiindexes $p, s \in \mathbb{N}^{n}, p \neq 0, s \neq 0$, and $p \perp s$,

$$
\begin{aligned}
\int_{\tau\left(\mathbb{B}_{n}\right)} & f_{p, s}(r) r^{2 \alpha+2 p^{*}-2 s^{*}+2 p}|r|^{-(|p|+|s|)} r d r \\
& \int_{\tau\left(\mathbb{B}_{n}\right)} \phi(r) r^{2 \alpha+2 p^{*}}|r|^{-\left(\left|p^{*}\right|+\left|s^{*}\right|\right)} r d r=0
\end{aligned}
$$

for all $\alpha \geq 2 s^{*}+s$. Next we will prove that either $\phi=0$ or $f_{p, s}=0$ for $p, s \in \mathbb{N}^{n}, p \neq 0, s \neq 0$, and $p \perp s$. Similarly as the proof of Theorem 19, we will prove it in the case of $n=2$. Let

$$
\begin{gathered}
E_{p, s}=\left\{\alpha \in \mathbb{Z}_{+}^{2}: \alpha \geq 2 s^{*}+s, \int_{\tau\left(\mathbb{B}_{n}\right)} f_{p, s}(r) r^{2 \alpha+2 p^{*}-2 s^{*}+2 p}\right. \\
\left.\times|r|^{-(|p|+|s|)} r d r=0\right\} .
\end{gathered}
$$

If $E_{p, s}$ satisfies condition (I), noting that

$$
\left.\left|f_{p, s}(r) r^{p+s}\right| r\right|^{-(|p|+|s|)}|=| \xi^{p} \bar{\xi}^{s} f_{p, s}(r) \mid<\infty,
$$

then $f_{p, s}=0$ by Lemma 10. Otherwise, let $E_{p, s}^{c}$ denote the complement of $E_{p, s}$ in $\left\{\alpha \in \mathbb{Z}_{+}^{2}: \alpha \succeq 2 s^{*}+s\right\}$; then $E_{p, s}^{c}$ satisfies condition (I) by Remark 6 . It follows from (52) that

$$
\int_{\tau\left(\mathbb{B}_{n}\right)} \phi(r) r^{2 \alpha+2 p^{*}}|r|^{-\left(\left|p^{*}\right|+\left|s^{*}\right|\right)} r d r=0, \quad \forall \alpha \in E_{p, s^{c}}^{c}
$$

Using Lemma 10 again, we obtain that $\phi=0$. Moreover, if either $f=0$ or $\phi=0$, then $T_{f} T_{g}\left(\bar{z}^{\alpha}\right)=0$. Hence, $T_{f} T_{g}=0$ implies that either $f=0$ or $\phi=0$.

The converse implication is obvious. This completes the proof.
Corollary 21. Let $f(z)=\xi^{p} \bar{\xi}^{s} f_{p, s}(r) \in L^{\infty}\left(\mathbb{B}_{n}, d V\right)$, where $p, s \in \mathbb{N}^{n}, p \neq 0, s \neq 0, p \perp s$, and $f_{p, s}(r) \in \mathscr{R}$. Then $T_{f}^{2}=T_{f}$ implies that either $f=0$ or $f=1$.

\section{The Commutativity of Toeplitz Operators with Quasihomogeneous and Separately Quasihomogeneous Symbols}

In this section, we will consider the commuting problem for two Toeplitz operators with certain symbols.

Theorem 22. Let $p, s \in \mathbb{N}^{n}$ be two nonzero multi-indexes with $p \perp s$ let $\psi \in \mathscr{R}$ such that $\xi^{p} \bar{\xi}^{s} \psi \in L^{\infty}\left(\mathbb{B}_{n}, d V\right)$, and let $\phi$ be a bounded radial function on $\mathbb{B}_{n}$. If $\phi$ is nonconstant, then $T_{\phi} T_{\xi^{p} \bar{\xi}_{\psi}^{s}}=T_{\xi^{p} \bar{\xi}_{\psi}^{s}} T_{\phi}$ if and only if either $|p|=|s|$ or $\psi=0$.

Proof. It follows from Lemmas 7 and 14 that $T_{\phi} T_{\xi^{p} \bar{\xi}^{s} \psi}=$ $T_{\xi^{p} \bar{\xi}_{\psi}^{s}} T_{\phi}$ if and only if

$$
\begin{aligned}
\int_{\tau\left(\mathbb{B}_{n}\right)} & \psi(r) r^{2 \alpha+2 p}|r|^{-(|p|+|s|)} r d r \\
& \times(2 n+2|\alpha|+2|p|-2|s|) \widehat{\phi} \\
& \times(2 n+2|\alpha|+2|p|-2|s|) \\
= & \int_{\tau\left(\mathbb{B}_{n}\right)} \psi(r) r^{2 \alpha+2 p}|r|^{-(|p|+|s|)} r d r \\
& \times(2 n+2|\alpha|) \widehat{\phi}(2 n+|\alpha|)
\end{aligned}
$$

for $p+\alpha \geq s$ and

$$
\begin{aligned}
\int_{\tau\left(\mathbb{B}_{n}\right)} & \psi(r) r^{2 \alpha+2 s}|r|^{-(|p|+|s|)} r d r \\
& \times(2 n+2|\alpha|+2|s|-2|p|) \widehat{\phi} \\
& \times(2 n+2|\alpha|+2|s|-2|p|) \\
= & \int_{\tau\left(\mathbb{B}_{n}\right)} \psi(r) r^{2 \alpha+2 s}|r|^{-(|p|+|s|)} r d r \\
& \times(2 n+2|\alpha|) \hat{\phi}(2 n+|\alpha|)
\end{aligned}
$$

for $s+\alpha \geq p$. As in the proof of Theorem 5.1 in [10], for $p+\alpha \geq$ $s,(56)$ and the property that $\phi$ is nonconstant imply that either $|p|=|s|$ or $\psi=0$. Furthermore, if either $|p|=|s|$ or $\psi=0$, it is easy to show that (57) holds for $s+\alpha \geq p$. Hence, $T_{\phi}$ commutes with $T_{\xi p} \bar{\xi}_{\psi}^{s}$ implies either $|p|=|s|$ or $\psi=0$.

Conversely, if either $|p|=|s|$ or $\psi=0$, one can get that (56) and (57) hold. That is, $T_{\phi} T_{\xi p \bar{\xi}^{s} \psi}=T_{\xi^{p} \bar{\xi}^{s} T_{\phi}} T_{\phi}$. This completes the proof.

As for $n=1$, a separately radial function is a radial and it follows that Toeplitz operators with separately radial symbols commute, there is no contradiction with an extension of a result in [29] to the case of $n \geq 2$. It is given in [29] that a Toeplitz operator on $L_{h}^{2}(\mathbb{D})$ with radial symbol commutes 
with another Toeplitz operator if that operator also has a radial symbol. The following theorem will show that this result is not true on $L_{h}^{2}\left(\mathbb{B}_{n}\right)(n \geq 2)$.

Theorem 23. For $n \geq 2$, let $\psi(z)$ be a bounded separately radial function and let $\phi(z)$ be a bounded radial function on $\mathbb{B}_{n}$. Then $T_{\psi}$ and $T_{\phi}$ commute.

Proof. The proof is similar to that of Theorem 22, so we omit it.

Next, we will give a description of commuting quasihomogeneous Toeplitz operators the with same degree.

Theorem 24. Let $p, s \in \mathbb{N}^{n}$ be two nonzero multi-indexes with $p \perp s,|p| \neq|s|$ and let $\phi, \psi$ be two square integrable radial function on $\mathbb{B}_{n}$ such that $\xi^{p} \bar{\xi}^{s} \phi, \xi^{p} \bar{\xi}^{s} \psi \in L^{\infty}\left(\mathbb{B}_{n}, d V\right)$. Then $T_{\xi^{p} \bar{\xi}_{\phi}^{s}} T_{\xi^{p} \bar{\xi}_{\psi}^{s}}=T_{\xi^{p} \bar{\xi}_{\psi}^{s} T_{\xi^{p} \bar{\xi}_{\phi}^{s}}}$ if and only if $\phi=C \psi$ for some constant $C$.

Proof. It follows from Lemma 8 that

$$
T_{\xi^{p} \bar{\xi}_{\phi}^{s}} T_{\xi^{p} \bar{\xi}^{s} \psi}=T_{\xi^{p} \bar{\xi}^{s} \psi} T_{\xi^{p} \bar{\xi}^{s} \phi}
$$

if and only if

$$
\begin{aligned}
\widehat{\phi}(2 n+ & 2|\alpha|+3|p|-3|s|) \widehat{\psi}(2 n+2|\alpha|+|p|-|s|) \\
= & \widehat{\psi}(2 n+2|\alpha|+3|p|-3|s|) \widehat{\phi} \\
& \times(2 n+2|\alpha|+|p|-|s|)
\end{aligned}
$$

for all $\alpha+2 p \succeq 2 s$ and

$$
\begin{aligned}
\widehat{\phi}(2 n+ & 2|\alpha|+3|s|-3|p|) \widehat{\psi}(2 n+2|\alpha|+|s|-|p|) \\
= & \widehat{\psi}(2 n+2|\alpha|+3|s|-3|p|) \widehat{\phi} \\
& \times(2 n+2|\alpha|+|s|-|p|)
\end{aligned}
$$

for all $\alpha+2 s \geq 2 p$. If $|p|>|s|$, according to Lemma 3, then (59) holds which implies that

$$
\begin{array}{r}
\widehat{\phi}(z+2|p|-2|s|) \widehat{\psi}(z)=\widehat{\psi}(z+2|p|-2|s|) \widehat{\phi}(z), \\
z \in\{z: \operatorname{Re} z>0\}
\end{array}
$$

It follows from Lemma 6 in [36] that $\phi=C \psi$. Furthermore, if $\phi=C \psi$, then (60) holds. Otherwise, if $|p|<|s|$, by a similar argument, (60) implies that $\phi=C \psi$ and so (59) holds.

The converse implication is obvious. This completes the proof.

Theorem 25. Let $f(z)=\sum_{s \in \mathbb{N}^{n}} \bar{\xi}^{s} f_{s}(r) \in L^{\infty}\left(\mathbb{B}_{n}, d V\right)$, where $f_{s}(r) \in \mathscr{R}$. Let $\phi$ be a bounded separately radial function on $\mathbb{B}_{n}$. Then $T_{f} T_{\phi}=T_{\phi} T_{f}$ if and only if $T_{\bar{\xi}^{s} f_{s}(r)} T_{\phi}=T_{\phi} T_{\bar{\xi}^{s} f_{s}(r)}$ for every multi-index $s \in \mathbb{N}^{n}$.
Proof. Using Lemmas 11 and 14, one can get that for any multiindex $\alpha \in \mathbb{N}^{n}$

$$
\begin{aligned}
& T_{f} T_{\phi}\left(z^{\alpha}\right)=\mu_{\alpha} \sum_{s \in \mathbb{N}^{n}} T_{\bar{\xi}^{s} f_{s}(r)}\left(z^{\alpha}\right) \\
& =\mu_{\alpha}\left[\sum_{s \in \mathbb{N}^{n}, \alpha \succeq s} T_{\bar{\xi}^{s} f_{s}(r)}\left(z^{\alpha}\right)\right. \\
& +\sum_{s \in \mathbb{N}^{n}, s \succeq \alpha, s \neq \alpha} T_{\bar{\xi}^{s} f_{s}(r)}\left(z^{\alpha}\right) \\
& \left.+\sum_{s \in \mathbb{N}^{n}, \alpha \nsucceq s, s \nsucceq \alpha} T_{\bar{\xi}^{s} f_{s}(r)}\left(z^{\alpha}\right)\right] \\
& =\mu_{\alpha}\left[\sum _ { s \in \mathbb { N } ^ { n } , \alpha \geq s } \left(\left(2^{n}(n+|\alpha|-|s|) !\right.\right.\right. \\
& \left.\times \int_{\mathcal{\tau}\left(\mathbb{B}_{n}\right)} f_{s}(r) r^{2 \alpha}|r|^{-|s|} r d r\right) \\
& \left.\times((\alpha-s) !)^{-1}\right) z^{\alpha-s} \\
& +\sum_{s \in \mathbb{N}^{n}, s \succeq \alpha, s \neq \alpha}\left(\left(2^{n}(n+|s|-|\alpha|) !\right.\right. \\
& \times \int_{\tau\left(\mathbb{B}_{n}\right)} f_{s}(r) r^{2 s} \\
& \left.\times|r|^{-|s|} r d r\right) \\
& \left.\left.\times((s-\alpha) !)^{-1}\right) \bar{z}^{s-\alpha}\right] \text {, }
\end{aligned}
$$

where $\mu_{\alpha}=2^{n}(n+|\alpha|) ! \int_{\tau\left(\mathbb{B}_{n}\right)} \phi(r) r^{2 \alpha} r d r / \alpha$ !. It follows that

$$
\begin{aligned}
& \left\langle T_{f} T_{\phi}\left(z^{\alpha}\right), z^{\beta}\right\rangle \\
& = \begin{cases}0 & \alpha \nsucceq \beta, \\
\frac{2^{n} \mu_{\alpha}(n+|\beta|) ! \int_{\tau\left(\mathbb{B}_{n}\right)} f_{\alpha-\beta}(r) r^{2 \alpha}|r|^{|\beta|-|\alpha|} r d r}{\beta !} & \\
\times\left\langle z^{\beta}, z^{\beta}\right\rangle & \alpha \geq \beta\end{cases}
\end{aligned}
$$

for $\beta \succeq 0$ and

$$
\begin{aligned}
& \left\langle T_{f} T_{\phi}\left(z^{\alpha}\right), \bar{z}^{\beta}\right\rangle \\
& =\frac{2^{n} \mu_{\alpha}(n+|\beta|) ! \int_{\tau\left(\mathbb{B}_{n}\right)} f_{\alpha+\beta}(r) r^{2 \alpha+2 \beta}|r|^{-(|\beta|+|\alpha|)} r d r}{\beta !} \\
& \quad \times\left\langle z^{\beta}, z^{\beta}\right\rangle
\end{aligned}
$$

for $\beta \succeq 0$ and $\beta \neq 0$. 
By a similar argument, one can deduce that

$$
\begin{aligned}
& \left\langle T_{\phi} T_{f}\left(z^{\alpha}\right), z^{\beta}\right\rangle \\
& = \begin{cases}0 & \alpha \nsucceq \beta, \\
\frac{2^{n} v_{\beta}(n+|\beta|) ! \int_{\tau\left(\mathbb{B}_{n}\right)} f_{\alpha-\beta}(r) r^{2 \alpha}|r|^{|\beta|-|\alpha|} r d r}{\beta !} & \\
\times\left\langle z^{\beta}, z^{\beta}\right\rangle & \alpha \geq \beta\end{cases}
\end{aligned}
$$

for $\beta \succeq 0$ and

$$
\begin{aligned}
& \left\langle T_{\phi} T_{f}\left(z^{\alpha}\right), \bar{z}^{\beta}\right\rangle \\
& =\frac{2^{n} v_{\beta}(n+|\beta|) ! \int_{\tau\left(\mathbb{B}_{n}\right)} f_{\alpha+\beta}(r) r^{2 \alpha+2 \beta}|r|^{-(|\beta|+|\alpha|)} r d r}{\beta !} \\
& \quad \times\left\langle z^{\beta}, z^{\beta}\right\rangle
\end{aligned}
$$

for $\beta \succeq 0$ and $\beta \neq 0$, where $\nu_{\beta}=2^{n}(n+|\beta|) ! \int_{\tau\left(\mathbb{B}_{n}\right)}$ $\phi(r) r^{2 \beta} r d r / \beta !$

Now suppose that $T_{f} T_{\phi}=T_{\phi} T_{f}$. It follows that $T_{f} T_{\phi}\left(z^{\alpha}\right)=T_{\phi} T_{f}\left(z^{\alpha}\right)$ and $T_{f} T_{\phi}\left(\bar{z}^{\alpha}\right)=T_{\phi} T_{f}\left(\bar{z}^{\alpha}\right)$ for any multi-index $\alpha \in \mathbb{N}^{n}$. Using Lemmas 11 and 14 again, we have

$$
\begin{aligned}
& \left\langle T_{\bar{\xi}^{s} f_{s}} T_{\phi}\left(z^{\alpha}\right), z^{\beta}\right\rangle \\
& =\left\{\begin{array}{cl}
\left(\left(2^{n} \mu_{\alpha}(n+|\beta|) !\right.\right. & \\
\quad \times \int_{\tau\left(\mathbb{B}_{n}\right)} f_{\alpha-\beta}(r) r^{2 \alpha} & \\
\left.\quad \times|r|^{|\beta|-|\alpha|} r d r\right) & \\
\left.\times(\beta !)^{-1}\right)\left\langle z^{\beta}, z^{\beta}\right\rangle & \beta=\alpha-s, \\
0 & \text { others }
\end{array}\right. \\
& = \begin{cases}\left\langle T_{f} T_{\phi}\left(z^{\alpha}\right), z^{\beta}\right\rangle & \beta=\alpha-s, \\
0 & \text { others }\end{cases} \\
& = \begin{cases}\left\langle T_{\phi} T_{f}\left(z^{\alpha}\right), z^{\beta}\right\rangle & \beta=\alpha-s, \\
0 & \text { others }\end{cases} \\
& =\left\{\begin{array}{cl}
\left(\left(2^{n} v_{\beta}(n+|\beta|) !\right.\right. & \\
\quad \times \int_{\mathcal{\tau}\left(\mathbb{B}_{n}\right)} f_{\alpha-\beta}(r) r^{2 \alpha} & \\
\left.\quad \times|r|^{|\beta|-|\alpha|} r d r\right) & \\
\left.\times(\beta !)^{-1}\right)\left\langle z^{\beta}, z^{\beta}\right\rangle & \beta=\alpha-s, \\
0 & \text { others }
\end{array}\right. \\
& =\left\langle T_{\phi} T_{\bar{\xi}^{s} f_{s}}\left(z^{\alpha}\right), z^{\beta}\right\rangle
\end{aligned}
$$

for any multi-indexes $\beta \in \mathbb{N}^{n}$ and $s \in \mathbb{N}^{n}$ and

$$
\begin{aligned}
& \left\langle T_{\bar{\xi}^{s} f_{s}} T_{\phi}\left(z^{\alpha}\right), \bar{z}^{\beta}\right\rangle \\
& =\left\{\begin{array}{cl}
\left(\left(2^{n} \mu_{\alpha}(n+|\beta|) !\right.\right. & \\
\quad \times \int_{\tau\left(\mathbb{B}_{n}\right)} f_{\alpha+\beta}(r) r^{2 \alpha+2 \beta} & \\
\left.\quad \times|r|^{-(|\beta|+|\alpha|)} r d r\right) & \\
\left.\quad \times(\beta !)^{-1}\right)\left\langle z^{\beta}, z^{\beta}\right\rangle & \beta=s-\alpha, \\
0 & \text { others }
\end{array}\right. \\
& = \begin{cases}\left\langle T_{f} T_{\phi}\left(z^{\alpha}\right), \bar{z}^{\beta}\right\rangle & \beta=s-\alpha, \\
0 & \text { others }\end{cases} \\
& = \begin{cases}\left\langle T_{\phi} T_{f}\left(z^{\alpha}\right), \bar{z}^{\beta}\right\rangle & \beta=s-\alpha, \\
0 & \text { others }\end{cases}
\end{aligned}
$$

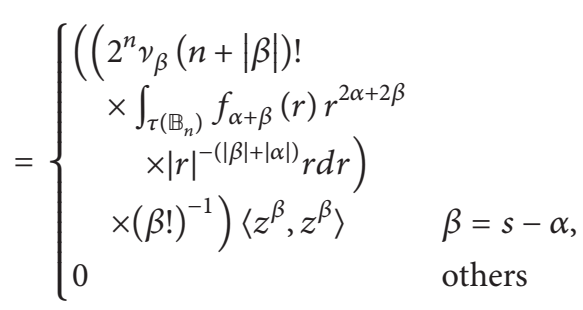

$$
\begin{aligned}
& =\left\langle T_{\phi} T_{\bar{\xi}^{s} f_{s}}\left(z^{\alpha}\right), \bar{z}^{\beta}\right\rangle
\end{aligned}
$$

for any multi-indexes $\beta \in \mathbb{N}^{n}, \beta \neq 0$, and $s \in \mathbb{N}^{n}$. By the same technique, we get that

$$
\left\langle T_{\bar{\xi}^{s} f_{s}} T_{\phi}\left(\bar{z}^{\alpha}\right), z^{\beta}\right\rangle=\left\langle T_{\bar{\xi}^{s} f_{s}} T_{\phi}\left(\bar{z}^{\alpha}\right), z^{\beta}\right\rangle
$$

for any multi-indexes $\beta \in \mathbb{N}^{n}$ and $s \in \mathbb{N}^{n}$, and

$$
\left\langle T_{\bar{\xi}^{s} f_{s}} T_{\phi}\left(\bar{z}^{\alpha}\right), \bar{z}^{\beta}\right\rangle=\left\langle T_{\bar{\xi}^{s} f_{s}} T_{\phi}\left(\bar{z}^{\alpha}\right), \bar{z}^{\beta}\right\rangle
$$

for any multi-indexes $\beta \in \mathbb{N}^{n}, \beta \neq 0$, and $s \in \mathbb{N}^{n}$. Hence $T_{\bar{\xi}^{s} f_{s}} T_{\phi}=T_{\phi} T_{\bar{\xi}^{s} f_{s}}$

Conversely, if $T_{\bar{\xi}^{s} f_{s}} T_{\phi}=T_{\phi} T_{\bar{\xi}^{s} f_{s}}$, it is easy to show that $T_{f} T_{\phi}=T_{\phi} T_{f}$. This completes the proof.

It is well known that $T_{\phi}^{*}=T_{\bar{\phi}}$, so one can easily get the following result.

Theorem 26. Let $f(z)=\sum_{p \in \mathbb{N}^{n}} \xi^{p} f_{p}(r) \in L^{\infty}\left(\mathbb{B}_{n}, d V\right)$, where $f_{p}(r) \in \mathscr{R}$. Let $\phi$ be a bounded separately radial function on $\mathbb{B}_{n}$. Then $T_{f} T_{\phi}=T_{\phi} T_{f}$ if and only if $T_{\xi^{p} f_{p}(r)} T_{\phi}=T_{\phi} T_{\xi^{p}} f_{p}(r)$ for every multi-index $p \in \mathbb{N}^{n}$.

\section{Acknowledgment}

This research is supported by the National Natural Science Foundation of China (Grant no. 11271059).

\section{References}

[1] W. Rudin, Function Theory in the Unit Ball of $\mathbb{C}^{n}$, Classics in Mathematics, Springer, Berlin, Germany, 2008. 
[2] A. Brown and P. R. Halmos, "Algebraic properties of Toeplitz operators," Journal für die Reine und Angewandte Mathematik, vol. 213, pp. 89-102, 1963/1964.

[3] P. Ahern and Ž. Čučković, "A theorem of Brown-Halmos type for Bergman space Toeplitz operators," Journal of Functional Analysis, vol. 187, no. 1, pp. 200-210, 2001.

[4] P. Ahern, "On the range of the Berezin transform," Journal of Functional Analysis, vol. 215, no. 1, pp. 206-216, 2004.

[5] I. Louhichi, E. Strouse, and L. Zakariasy, "Products of Toeplitz operators on the Bergman space," Integral Equations and Operator Theory, vol. 54, no. 4, pp. 525-539, 2006.

[6] X.-T. Dong and Z.-H. Zhou, "Products of Toeplitz operators on the harmonic Bergman space," Proceedings of the American Mathematical Society, vol. 138, no. 5, pp. 1765-1773, 2010.

[7] X. Ding, "Products of Toeplitz operators on the polydisk," Integral Equations and Operator Theory, vol. 45, no. 4, pp. 389403, 2003.

[8] B. R. Choe, Y. J. Lee, K. Nam, and D. Zheng, "Products of Bergman space Toeplitz operators on the polydisk," Mathematische Annalen, vol. 337, no. 2, pp. 295-316, 2007.

[9] Z.-H. Zhou and X.-T. Dong, "Algebraic properties of Toeplitz operators with radial symbols on the Bergman space of the unit ball," Integral Equations and Operator Theory, vol. 64, no. 1, pp. 137-154, 2009.

[10] X.-T. Dong and Z.-H. Zhou, "Algebraic properties of Toeplitz operators with separately quasihomogeneous symbols on the Bergman space of the unit ball," Journal of Operator Theory, vol. 66, no. 1, pp. 193-207, 2011.

[11] B. Zhang and Y. Lu, "Toeplitz operators with quasihomogeneous symbols on the Bergman space of the unit ball," Journal of Function Spaces and Applications, vol. 2012, Article ID 414201, 16 pages, 2012.

[12] J. Yang, L. Liu, and Y. Lu, "Algebraic properties of Toeplitz operators on the pluriharmonic Bergman space," Journal of Function Spaces and Applications, vol. 2013, Article ID 578436, 12 pages, 2013.

[13] K. Y. Guo, "A problem on products of Toeplitz operators," Proceedings of the American Mathematical Society, vol. 124, no. 3, pp. 869-871, 1996.

[14] C. Gu, "Products of several Toeplitz operators," Journal of Functional Analysis, vol. 171, no. 2, pp. 483-527, 2000.

[15] A. Aleman and D. Vukotić, "Zero products of Toeplitz operators," Duke Mathematical Journal, vol. 148, no. 3, pp. 373-403, 2009.

[16] P. Ahern and Ž. Čučković, "Some examples related to the Brown-Halmos theorem for the Bergman space," Acta Universitatis Szegediensis, vol. 70, no. 1-2, pp. 373-378, 2004.

[17] Ž. Čučković, "Berezin versus Mellin," Journal of Mathematical Analysis and Applications, vol. 287, no. 1, pp. 234-243, 2003.

[18] I. Louhichi, N. V. Rao, and A. Yousef, "Two questions on products of Toeplitz operators on the Bergman space," Complex Analysis and Operator Theory, vol. 3, no. 4, pp. 881-889, 2009.

[19] W. Bauer and N. Vasilevski, "On the structure of a commutative Banach algebra generated By Toeplitz operators with quasiradial quasi-homogeneous symbols," Integral Equations and Operator Theory, vol. 74, no. 2, pp. 199-231, 2012.

[20] S. Axler and Ž. Čučković, "Commuting Toeplitz operators with harmonic symbols," Integral Equations and Operator Theory, vol. 14, no. 1, pp. 1-12, 1991.

[21] Ž. Čučković and N. V. Rao, "Mellin transform, monomial symbols, and commuting Toeplitz operators," Journal of Functional Analysis, vol. 154, no. 1, pp. 195-214, 1998.
[22] I. Louhichi and L. Zakariasy, "On Toeplitz operators with quasihomogeneous symbols," Archiv der Mathematik, vol. 85, no. 3, pp. 248-257, 2005.

[23] D. Zheng, "Commuting Toeplitz operators with pluriharmonic symbols," Transactions of the American Mathematical Society, vol. 350, no. 4, pp. 1595-1618, 1998.

[24] N. Vasilevski, "Toeplitz operator on the Bergman spaces: insidethe-Domain Effects," Contemporary Mathematics, vol. 289, pp. 79-146, 2001.

[25] N. L. Vasilevski, "Bergman space structure, commutative algebras of Toeplitz operators, and hyperbolic geometry," Integral Equations and Operator Theory, vol. 46, no. 2, pp. 235-251, 2003.

[26] R. Quiroga-Barranco and N. Vasilevski, "Commutative $C^{*}$ algebras of Toeplitz operators on the unit ball. I. Bargmann-type transforms and spectral representations of Toeplitz operators," Integral Equations and Operator Theory, vol. 59, no. 3, pp. 379419, 2007.

[27] R. Quiroga-Barranco and N. Vasilevski, "Commutative $C^{*}$ algebras of Toeplitz operators on the unit ball. II. Geometry of the level sets of symbols," Integral Equations and Operator Theory, vol. 60, no. 1, pp. 89-132, 2008.

[28] X. Dong and Z. Zhou, "Commuting quasi-homogeneous Toeplitz operators on the harmonic Bergman space," Complex Analysis and Operator Theory, vol. 7, no. 4, pp. 1267-1285, 2012.

[29] I. Louhichi and L. Zakariasy, "Quasihomogeneous Toeplitz operators on the harmonic Bergman space," Archiv der Mathematik, vol. 98, no. 1, pp. 49-60, 2012.

[30] W. Bauer and N. Vasilevski, "Banach algebras of commuting Toeplitz operators on the unit ball via the quasi-hyperbolic group," in A Panorama of Modern Operator Theory and Related Topics, vol. 218 of Operator Theory: Advances and Applications, pp. 155-175, Springer, Berlin, Germany, 2012.

[31] W. Bauer and N. Vasilevski, "Commutative Toeplitz Banach algebras on the ball and quasi-nilpotent group action," Integral Equations and Operator Theory, vol. 72, no. 2, pp. 223-240, 2012.

[32] N. Vasilevski, "Quasi-radial quasi-homogeneous symbols and commutative Banach algebras of Toeplitz operators," Integral Equations and Operator Theory, vol. 66, no. 1, pp. 141-152, 2010.

[33] N. Vasilevski, "Parabolic quasi-radial quasi-homogeneous symbols and commutative algebras of Toeplitz operators," in Topics in Operator Theory, vol. 202 of Operator Theory: Advances and Applications, pp. 553-568, Birkhäuser, Basel, Switzerland, 2010.

[34] R. Quiroga-Barranco and N. Vasilevski, "Commutative algebras of Toeplitz operators on the Reinhardt domains," Integral Equations and Operator Theory, vol. 59, no. 1, pp. 67-98, 2007.

[35] R. Remmert, Classical Topics in Complex Function Theory, vol. 172 of Graduate Texts in Mathematics, Springer, New York, NY, USA, 1998.

[36] I. Louhichi, "Powers and roots of Toeplitz operators," Proceedings of the American Mathematical Society, vol. 135, no. 5, pp. 1465-1475, 2007. 


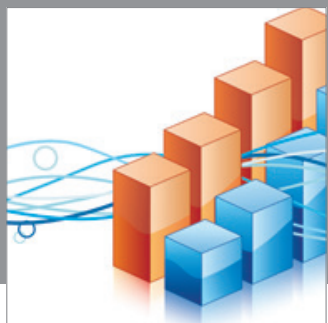

Advances in

Operations Research

mansans

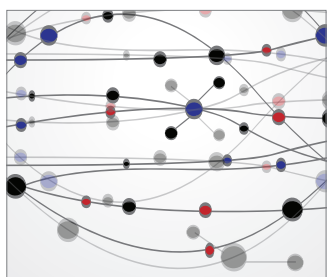

The Scientific World Journal
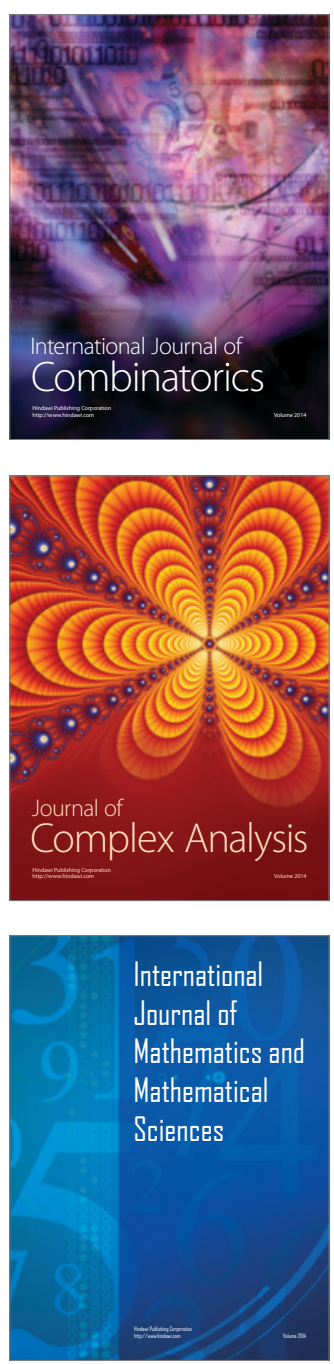
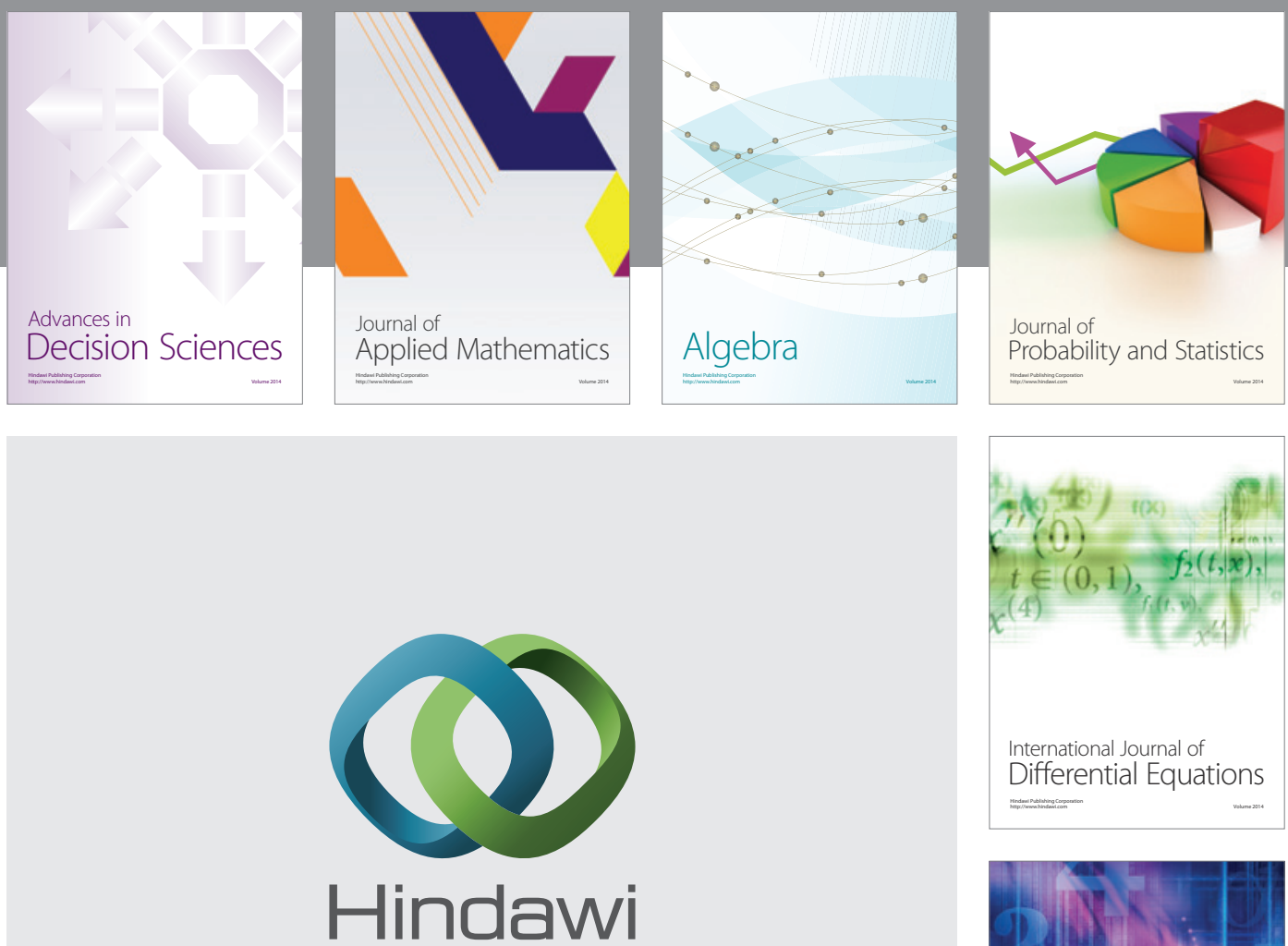

Submit your manuscripts at http://www.hindawi.com
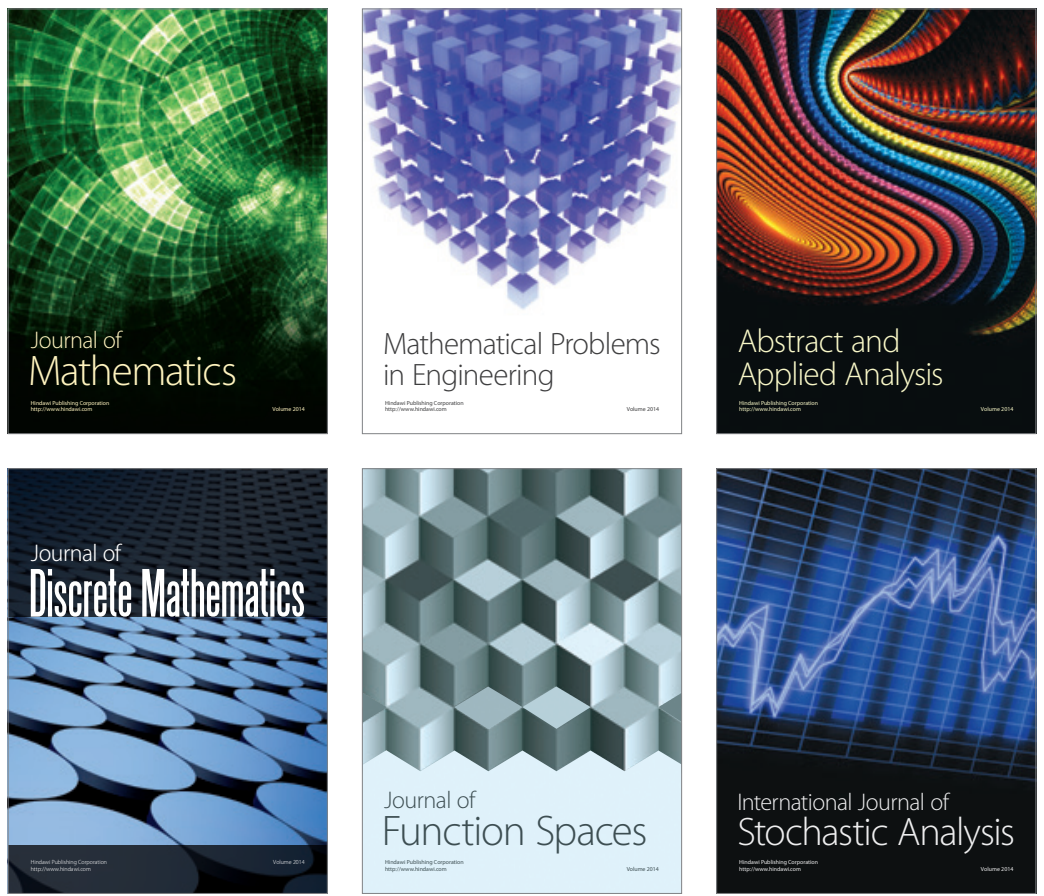

Journal of

Function Spaces

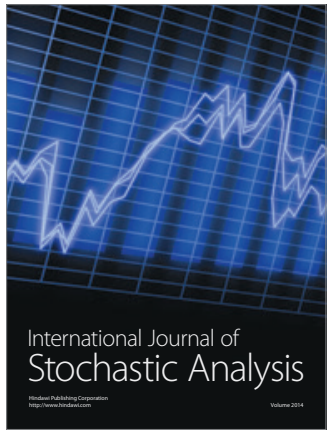

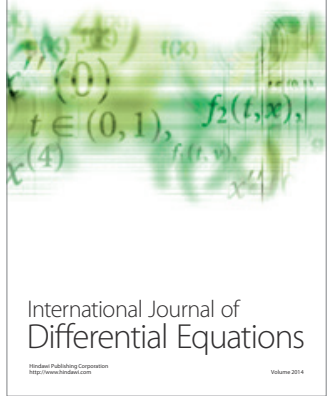
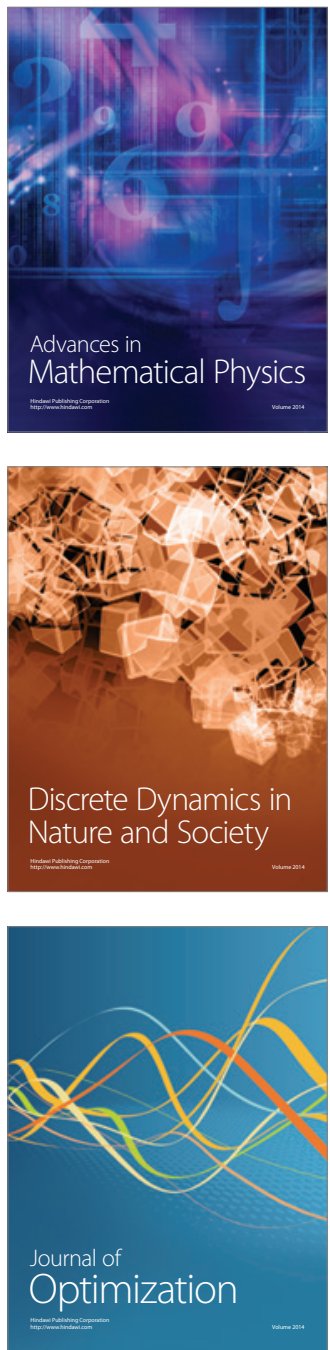